\title{
A general mechanism for signal propagation in the nicotinic acetylcholine receptor family
}

Ana Sofia F. Oliveira, ${ }^{\mathrm{a}, \mathrm{b}}$ Christopher J. Edsall ${ }^{\mathrm{c}}$, Christopher J. Woods, ${ }^{\mathrm{b}, \mathrm{c}}$ Phil Bates, ${ }^{\mathrm{d}, \mathrm{e}}$ Gerardo Viedma Nunez, ${ }^{e}$ Susan Wonnacott, ${ }^{f}$ Isabel Bermudez, ${ }^{\mathrm{g}}$ Giovanni Ciccotti, ${ }^{\mathrm{h}, \mathrm{i} j}$ Timothy C. Gallagher, ${ }^{b}$ Richard B. Sessions ${ }^{\mathrm{a}^{*}}$ and Adrian J. Mulholland ${ }^{\mathrm{b}^{*}}$

*E-mail: r.sessions@bristol.ac.uk adrian.mulholland@bristol.ac.uk

${ }^{\text {a }}$ School of Biochemistry, University of Bristol, Bristol BS8 1DT (United Kingdom)

${ }^{\mathrm{b}}$ Centre for Computational Chemistry, School of Chemistry, University of Bristol, Bristol BS8 1TS (United Kingdom)

${ }^{c}$ Research Software Engineering, Advanced Computing Research Centre, University of Bristol, Bristol BS1 5QD (United Kingdom)

${ }^{\mathrm{d}}$ Department of Computer Science, Faculty of Engineering, University of Bristol, Bristol BS8 1TR (United Kingdom)

${ }^{\mathrm{e}}$ Oracle Corporation, Oracle Cloud Development Centre, Bristol BS2 2JJ (United Kingdom)

${ }^{\mathrm{f}}$ Department of Biology and Biochemistry, University of Bath, Bath BA2 7AY (United Kingdom)

g Department of Biological and Medical Sciences, Oxford Brookes University, Oxford OX30BP, UK

h Institute for Applied Computing "Mauro Picone" (IAC), CNR, Via dei Taurini 19, 00185 Rome, Italy

${ }^{i}$ School of Physics, University College of Dublin UCD-Belfield, Dublin 4, Ireland

${ }^{j}$ Università di Roma La Sapienza, Ple. A. Moro 5, 00185 Roma, Italy

\begin{abstract}
Nicotinic acetylcholine receptors (nAChRs) modulate synaptic activity in the central nervous system. The $\alpha 7$ subtype, in particular, has attracted considerable interest in drug discovery as a target for several conditions, including Alzheimer's disease and schizophrenia. Identifying agonist-induced structural changes underlying $\mathrm{nAChR}$ activation is fundamentally important for understanding biological function and rational drug design. Here, extensive equilibrium and nonequilibrium molecular dynamics simulations, enabled by cloud-based highperformance computing, reveal the molecular mechanism by which structural changes induced by agonist unbinding are transmitted within the human $\alpha 7 \mathrm{nAChR}$. The simulations reveal the sequence of coupled structural changes involved in driving conformational change responsible for biological function. Comparison with simulations of the $\alpha 4 \beta 2 \mathrm{nAChR}$ subtype
\end{abstract}


identifies features of the dynamical architecture common to both receptors, suggesting a general structural mechanism for signal propagation in this important family of receptors.

\section{Main text}

Nicotinic acetylcholine receptors (nAChR) are prototypical members of the Cys loop pentameric ligand-gated ion channel (pLGICs) family, which also includes the $\mathrm{GABA}_{\mathrm{A}}$ and 5-HT3 receptors. ${ }^{1-3}$ In the peripheral nervous system, nAChRs mediate fast excitatory synaptic signalling whereas in the brain they mostly modulate the synaptic signalling of a wide range of neurotransmitters. ${ }^{4}$ Neuronal nAChRs expressed in the CNS are putative targets for the treatment of a variety of neurodegenerative diseases, neurodevelopmental disorders, pain and addiction ${ }^{5}$, and analogous nAChRs in the insect CNS are targets for neonicotinoid pesticides. ${ }^{6}$ The $\alpha 7$ subtype is one of most abundant nAChRs subtypes in the mammalian $\mathrm{CNS}^{5}$, attracting considerable interest for drug discovery due to its role in cognition, attention, memory and sensory processing. ${ }^{7} \alpha 7 \mathrm{nAChR}$ dysfunction is implicated in disorders such as Alzheimer's and Parkinson's diseases and schizophrenia. ${ }^{5}$

There are many nAChR subtypes, distinguished by their specific combination of five subunits ${ }^{1-3,8}$. Despite differences in sequence (Figure S1), all subunits share the same basic architecture (Figure 1), consisting of a N-terminal extracellular domain (ECD), a transmembrane domain (TMD), a variable cytoplasmic domain (ICD) and a short extracellular C-terminal domain. ${ }^{1-3}$ The structures of nAChRs (and other pLGICs) have been revealed by cryo-electron microscopy ${ }^{9-11}$ and X-ray crystallography ${ }^{12}$. The agonist-binding pockets are located in the ECDs at the interface between two neighbouring subunits (Figure 1). The $\alpha 7$ receptor subtype is unusual in being formed of five identical $\alpha 7$ subunits symmetrically arranged around a central ion channel ${ }^{1-3}$ and thus presents five equivalent binding pockets, lined by several highly conserved aromatic residues. ${ }^{13}$

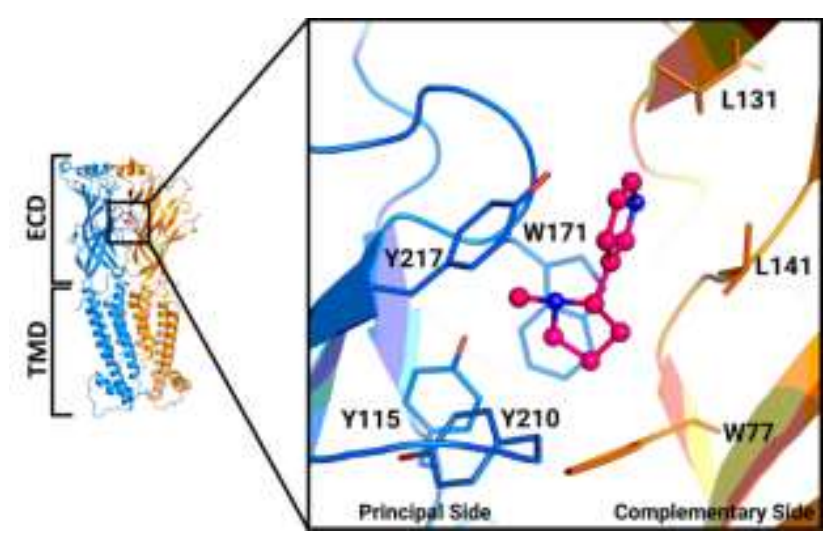

Figure 1. Close-up view of the ligand-binding pocket of human $\alpha 7 \mathbf{n A C h R}$. A model for the $\alpha 7$ subtype was built using as a template the $\alpha 4$ subunit of the human $\alpha 4 \beta 2 \mathrm{nAChR}{ }^{12}$. Nicotine was modelled in two nonconsecutive binding pockets, similar to what is observed in the human $\alpha 4 \beta 2 \mathrm{nAChR}$ structure $^{12}$ (see Supporting Information). Nicotine is represented in balls-and-sticks. 
Binding of acetylcholine (and other agonists such as nicotine) leads to opening of the ion channel, causing a flow of positive ions across the membrane, triggering depolarisation and signalling mechanisms. ${ }^{2,14}$ Several regions at ECD-TMD interface have been shown to be essential for linking the agonist binding site to the channel gate (e.g. ${ }^{15-25}$ ), namely the $\beta 10$ M1 region (which covalently links the ECD and TMD) and the Cys, F and $\beta 1-\beta 2$ loops, which are in direct contact with the M2-M3 linker, a well-established gating control element. ${ }^{20,22,26-30}$ However, while the application of a variety of experimental approaches has led to a greater insight into the function of $\mathrm{nAChR}$, the conformational changes induced by agonist binding/unbinding and how those are communicated to the ion channel remain poorly defined. Answering this question requires knowledge of the dynamics of the protein and the temporal evolution of the conformational changes that take place upon ligand (un)binding. Biomolecular simulations have the potential to investigate these questions ${ }^{31}$, but face serious challenges associated with the large size of the systems and timescales involved; we show here that these can be overcome by the use of cloud-based high-performance computing (HPC) and by advanced, nonequilibrium molecular dynamics (MD) simulations, complementing standard equilibrium MD. In previous work on human $\alpha 4 \beta 2 \mathrm{nAChR}^{32}$, we demonstrated how a combination of equilibrium and nonequilibrium MD simulations identified the structural motifs involved in signal propagation upon nicotine unbinding and the sequence of the events associated with the first steps of this process. Here, we have applied this novel approach to the human $\alpha 7 \mathrm{nAChR}$, using cloud-based nonequilibrium simulations to complement more traditional high-performance computing to achieve long timescales and extensive sampling. We identify the dynamic structural mechanism of receptor response to nicotine and, by comparison with the human $\alpha 4 \beta 2 \mathrm{nAChR}$, we find a common signal propagation pathway in nAChRs, which may generally apply to pLGICs.

Extensive equilibrium MD simulations (totalling $10 \mu \mathrm{s}$ ), with and without nicotine bound, were performed to identify the conformational changes induced by the ligand in the human $\alpha 7 \mathrm{nAChR}$ receptor. These simulations show that nicotine induces conformational changes in the binding pocket region, namely in loop $\mathrm{B}$ and $\mathrm{C}$ and also at the ECD:TMD interface, namely in the Cys and F loops and in the M2-M3 linker (Figures S10-S13). These results correlate well with the experimental evidence indicating that loop $\mathrm{B}, \mathrm{C}$ and $\mathrm{F}$ have a role in binding (e.g. ${ }^{33,34}$ ) and agonist affinity ${ }^{35,36}$. Structural changes are also observed in the second layer of residues surrounding the binding site, mainly in the extracellular selectivity filter region (Figure S14). Note that a despite some differences in amplitude, similar structural changes are observed between the two binding pockets (Figure S10-S11). Although loop F has been shown to be essential for binding ${ }^{13}$, its role in signal propagation remains elusive. ${ }^{37,38}$ In our nicotine-bound equilibrium simulations, the motions of the upper part of loop F (residues D186-Y190) are coupled to the movements of the ECDs (right-side panels in Figure S15-S16), mostly to the binding-site region (loops B, C and loop D). The dynamics of the lower part of loop F (residues P192-W196) are highly correlated with the ECDs (namely the loops A, B, C and D in the binding pockets and the Cys loop at the interface between domains) and the TMDs (namely transmembrane helices M1, M2 and M3 and the M2-M3 linker).

When nicotine is present in the binding pockets, the dynamics of the M2-M3 linker is highly correlated not only with transmembrane helices 1, 2 and 3 but also with the Cys loop and some of the structural motifs forming the binding pockets, namely loops A, B and C (Figure S17). Several experimental studies have shown that mutations in the M2-M3 linker alter channel gating and disrupt the communication between domains in nAChRs (e.g. ${ }^{20,22,26,28-}$ $30,39,40$ ) and in other pLGICs (e.g. ${ }^{27,41-45}$ ). 
These simulations of human $\alpha 7 \mathrm{nAChR}$, combined with results for the human $\alpha 4 \beta 2 \mathrm{nAChR}^{32}$, reveal a common pattern of nicotine-induced structural rearrangements. Despite the differences in sequences between the two receptors, including differences in the Cys, B and C loops and M2-M3 linker (Figures S1-S2), similar conformational changes are observed in these structural elements. The largest difference in behaviour between the subtypes occurs in loop A, which shows a significant rearrangement in $\alpha 7$ but not in the $\alpha 4 \beta 2$ subtype.

Here, a set of 450 nonequilibrium simulations without nicotine was performed to identify the signal propagation pathway in the $\alpha 7 \mathrm{nAChR}$. The equilibrium and nonequilibrium simulations are complementary approaches. The former allows for identification of the agonist-induced conformational changes (after hundreds of nanoseconds), while the latter allows for the determination of the order of the events associated with signal propagation and interdomain communication.

In all nonequilibrium simulations performed, both nicotine molecules were instantaneously annihilated. These simulations reveal the response of the system to this perturbation, specifically showing the mechanical and dynamical coupling between structural elements involved in the response (Figures 2, S20-S22 and Movie 1). Note that the nonequilibrium simulations performed here do not imply free energy calculations (e.g. ${ }^{46-49}$ ). In this case, it is the introduction of a perturbation that forces the system out of equilibrium. The KuboOnsager approach pioneered by $\mathrm{Ciccotti}^{50-52}$ was used to compute the response of the receptor to nicotine removal (Figures S18-S19), by comparing the differences in the evolution of the simulations with and without nicotine, averaged over large numbers of simulations. The subtraction approach, and averaging over multiple (450) short simulations, allows conformational changes and their temporal sequence to be identified and their statistical significance to be determined. These nonequilibrium simulations are not intended to model the physical process of ligand (un)binding, nor the transition between states and, due to the artificial nature of the perturbation, the timescales observed for the response of the receptor do not represent the biological timescales. ${ }^{32}$ It is also important to note that, due to the short timescale of the nonequilibrium simulations ( $5 \mathrm{~ns}$ ), the observed deviations reflect only the first steps in the interdomain communication mechanism (Figures S20-S21). 


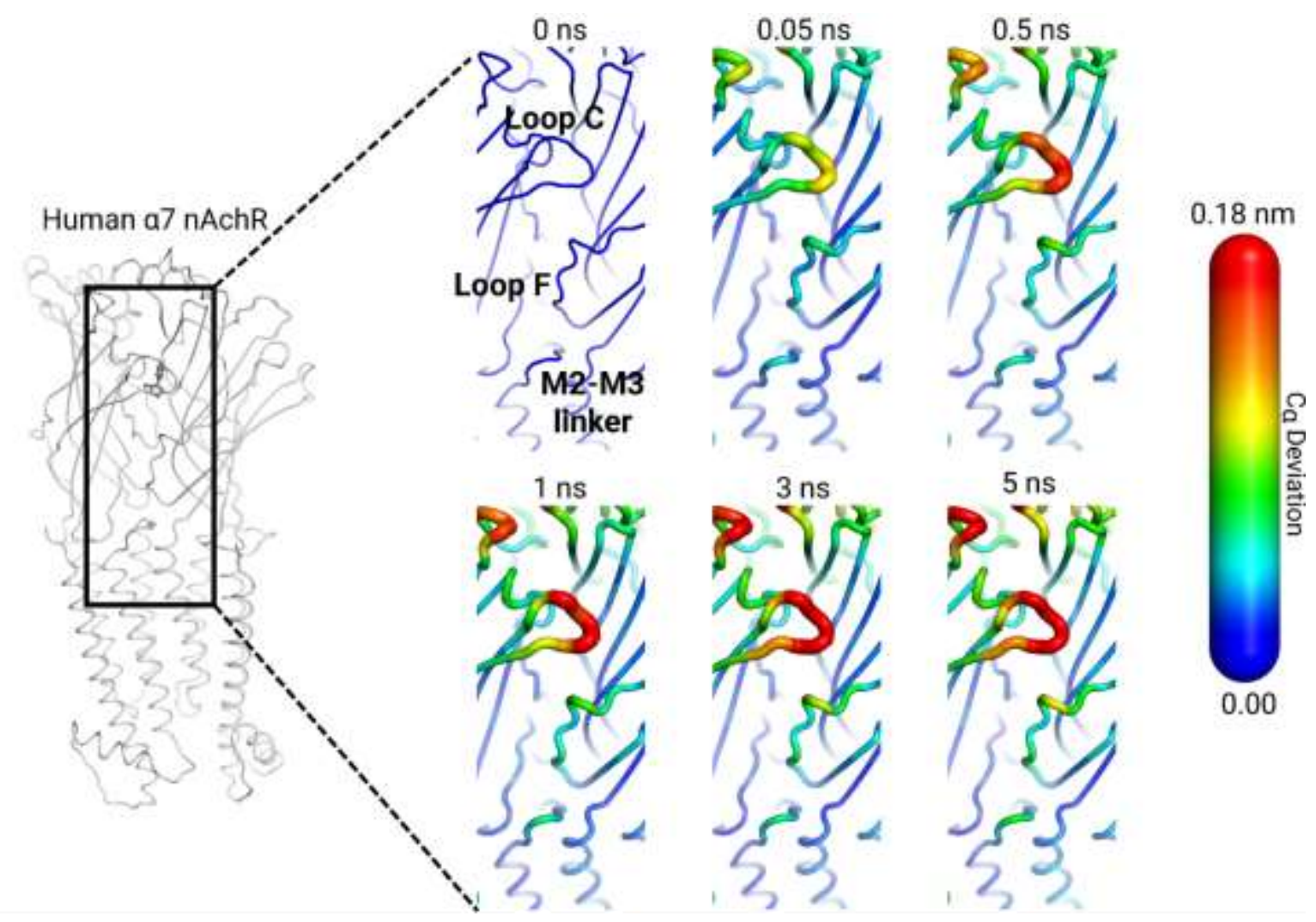

Figure 2- Signal propagation pathway from the ECD to the TMD in the $\alpha 7$ nAChR. Average C $\alpha$-positional deviation at times $0,0.05,0.5,1,3$ and $5 \mathrm{~ns}$ following nicotine annihilation from the first binding pocket. The $\mathrm{C} \alpha$ deviations between the simulations with and without nicotine were determined for each residue, and the final values were averaged over the 450 pairs of simulations (Figure S18-S19). The C $\alpha$ average deviations are mapped onto the average structure for the system without nicotine using the colour scheme in the scale on the right.

The 450 nonequilibrium simulations were performed in five days using the Oracle Cloud and 100 compute instances managed via cluster-in-the-cloud (https://cluster-in-thecloud.readthedocs.io and https://doi.org/10.5281/zenodo.3246253). Profiling cloud versus onpremises computing suggested that these simulations would have taken around three months on shared local HPC resources. This demonstrates the rapid turnaround and throughput of high-end scientific computation (in this case intensive, physics-based atomistic MD simulations) that is now possible using such cloud services. The approach here combines strengths of traditional HPC (for equilibrium simulations) with the cloud (for nonequilibirum simulations).

The nonequilibrium simulations show that signal transmission in the $\alpha 7$ receptor starts in the binding pocket region, in loop $\mathrm{C}$, and it then propagates to loop $\mathrm{F}$ and finally to the TMDs (Figure 2). Unsurprisingly, loop C (residues S206-Y217) is the first region to respond to ligand removal, and after $0.05 \mathrm{~ns}$, some conformational rearrangements are already observed in this region. Over the next few nanoseconds, gradual and cumulative conformational changes propagate to the top (residues D186-Y190) and then to the lower part of loop F (residues P192-W196) and afterwards to the TMDs via the M2-M3 linker (residues T286V290) (Figures 2, S20-S21 and Movie 1). Note that the two binding pockets show similar responses to nicotine annihilation, with the same order of events observed for each (Figures S18-S19). All the structural motifs identified here have been shown experimentally to be 
involved in ligand binding and signal transduction: Loop C is important for binding (e.g. ${ }^{33,34}$ ), contributing to binding the ammonium group of the agonists ${ }^{13,53}$; Loop $\mathrm{F}$ plays a role in ligand binding affinity, and specificity ${ }^{13}$; and the M2-M3 linker is essential for channel gating and communication between domains (e.g. ${ }^{22,26-30,39-45}$ ), and insertions/deletions of residues in this region directly affect the open-channel lifetime. ${ }^{30}$

\section{Signal propagation in nAChRs}

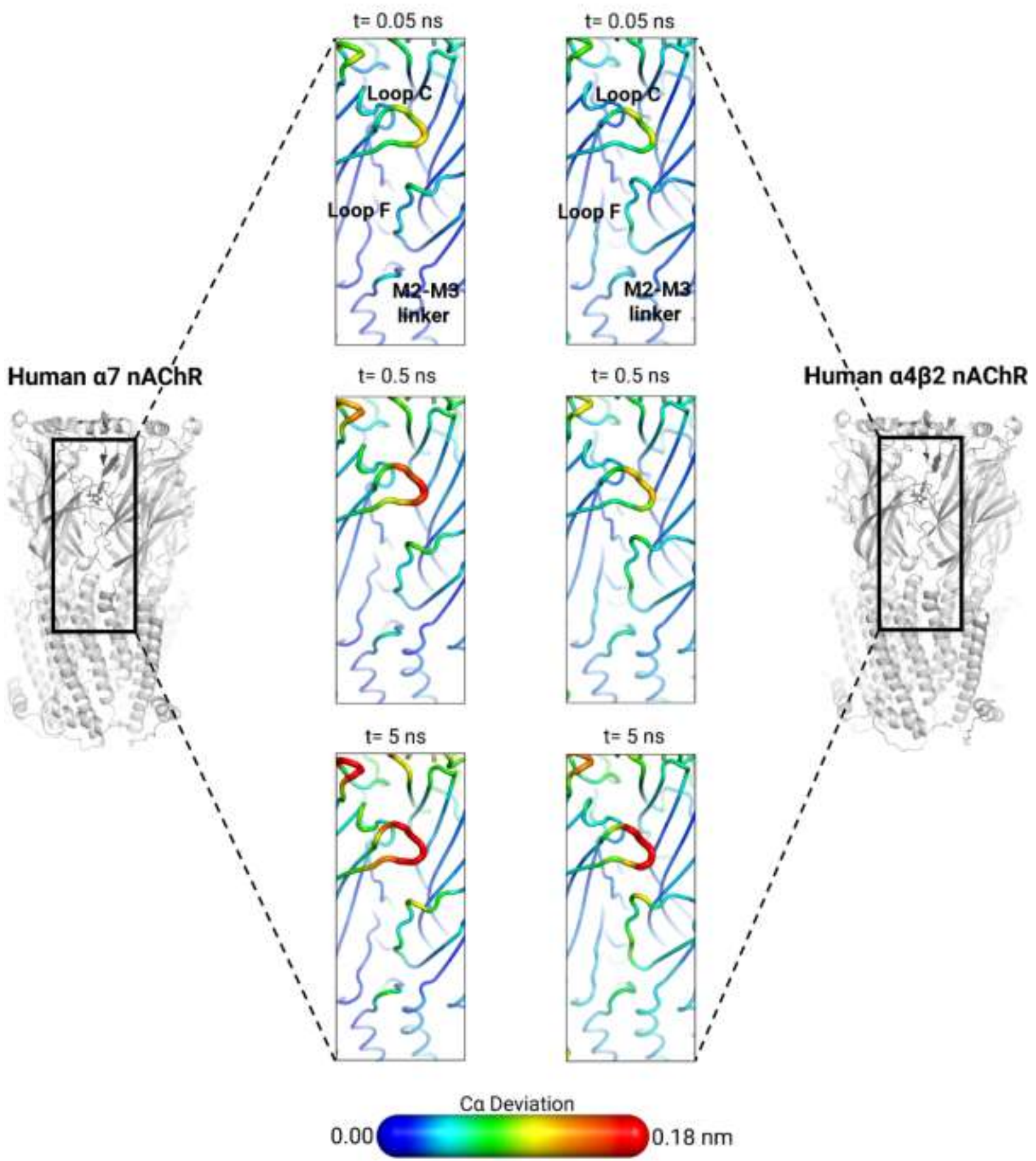

Figure 3- The ECD:TMD signal propagation pathway in human $\alpha 7$ and $\alpha 4 \beta 2$ nAChRs. The deviations for the $\alpha 4 \beta 2 \mathrm{nAChR}$ are taken from ${ }^{32}$. Note that although the apparent rate of propagation is different, the sequence of conformational changes associated with the initial steps of signal 
propagation is the same for both subtypes, i.e. the structural elements involved (loops C, F and M2M3 linker), and the sequence of structural changes, are the same.

The signal propagation pathway observed here for the $\alpha 7 \mathrm{nAChR}$ is remarkably similar to that of the $\alpha 4 \beta 2$ nAChR. ${ }^{32}$ The structural motifs involved in the signal transmission and the sequence of changes are the same between the two subtypes (Figure 3 and Movie 2). This supports the idea that, despite differences in sequence ${ }^{13,54}$, all family members share a common communication mechanism. Our simulations show differences in the rate of propagation, which may relate to differences in function and response between receptor subtypes. All known pLGICs have a similar molecular architecture ${ }^{1-3}$ and it has been shown experimentally that all of the structural elements identified here (loops C, F, Cys and the M2M3 linker) are important not only in nAChR (e.g. ${ }^{15,20,22,26,28,30,39,40}$ ) but also in other homologous receptors (e.g. ${ }^{37,38,55-58}$ ). Furthermore, it is also known that chimeric pLGICs formed by modular combinations of different ECDs and TMDs are still functional. ${ }^{21,59,60}$

Our findings identify a general mechanism for communication within this receptor family: the structural rearrangements associated with signal propagation start in loop $\mathrm{C}$ and are subsequently transmitted, gradually and cumulatively, to loop F, and then to the TMDs via the M2-M3 linker. This mechanism is consistent with experimental data and provides a molecular-level rationalisation of those data. This dynamic mechanism of signal propagation not only confirms the involvement of specific structural motifs but also shows, for the first time, the complex contribution of Loop F to signal propagation. It should also assist in the design of agonists or allosteric modulators to target nAChRs and other biomedically relevant pLGICs. The approach used here, combining extensive equilibrium and nonequilibrium simulations, is a valuable tool to study conformational changes in allosteric proteins.

\section{Acknowledgements}

We thank EPSRC (EP/N024117/1, EP/N018591/1 and EP/M022609/1) for support. The equilibrium simulations were carried out using the computational facilities of the Advanced Computing Research Centre, University of Bristol (http://www.bris.ac.uk/acrc). All nonequilibrium simulations were performed in the Oracle Public Cloud Infrastructure (https://cloud.oracle.com/en US/iaas). This work received generous support from the Oracle Cloud Innovation Accelerator (https://www.oracle.com/industries/education-andresearch/innovation-accelerator/) to enable data processing and molecular dynamics simulations using Oracle's high-performance public cloud infrastructure (https://cloud.oracle.com/en_US/cloud-infrastructure). We also thank Achieve Life Sciences for useful discussion and support.

\section{Author Contributions}

Conceptualization/design of the work: Ana Sofia F. Oliveira, Richard B. Sessions and Adrian J. Mulholland; Software creation: Ana Sofia F. Oliveira, Christopher J. Edsall, Christopher J. Woods, Phil Bates and Gerardo Viedma Nunez; Acquisition and analysis of the data: Ana Sofia F. Oliveira, Susan Wonnacott, Isabel Bermudez, Giovanni Ciccotti, Timothy C. Gallagher, Richard B. Sessions and Adrian J. Mulholland; Writing of the manuscript: Ana Sofia F. Oliveira, Richard B. Sessions and Adrian J. Mulholland; Review \& Editing of the 
manuscript: Christopher J. Woods, Phil Bates, Susan Wonnacott, Isabel Bermudez, Giovanni Ciccotti, Timothy C. Gallagher; Funding Acquisition: Phil Bates, Richard B. Sessions, Adrian J. Mulholland and Timothy C. Gallagher.

\section{References}

1. Thompson, A. J.; Lester, H. A.; Lummis, S. C., The structural basis of function in Cys-loop receptors. Q. Rev. Biophys. 2010, 43, 449-499.

2. Nemecz, A.; Prevost, M. S.; Menny, A.; Corringer, P. J., Emerging Molecular Mechanisms of Signal Transduction in Pentameric Ligand-Gated Ion Channels. Neuron 2016, 90, 452-470.

3. Changeux, J. P., The nicotinic acetylcholine receptor: a typical 'allosteric machine'. Philos. Trans. R. Soc. Lond. B Biol. Sci. 2018, 373.

4. Wonnacott, S., Presynaptic nicotinic ACh receptors. Trends in Neurosci. 1997, 20 (2), 92-98.

5. Dineley, K. T.; Pandya, A. A.; Yakel, J. L., Nicotinic ACh receptors as therapeutic targets in CNS disorders. Trends Pharmacol. Sci. 2015, 36, 96-108.

6. Casida, J.; Berenbaum, M., Neonicotinoids and Other Insect Nicotinic Receptor Competitive Modulators: Progress and Prospects. Annu. Rev. Entomol. 2018, 63, 125-144.

7. Haydar, S.; Dunlop, J., Neuronal Nicotinic Acetylcholine Receptors - Targets for the Development of Drugs to Treat Cognitive Impairment Associated with Schizophrenia and Alzheimer's Disease. Cur. Top. Med. Chem. 2010, 10 (2), 144-152.

8. Jones, A. S., DB, Diversity of Insect Nicotinic Acetylcholine Receptor Subunits. In Insect Nicotinic Acetylcholine Receptors. Advances in Experimental Medicine and Biology, Thany, S., Ed. Springer: New York, 2010; Vol. 683.

9. Miyazawa, A.; Fujiyoshi, Y.; Unwin, N., Structure and gating mechanism of the acetylcholine receptor pore. Nature 2003, 423, 949-55.

10. Unwin, N., Nicotinic acetylcholine receptor and the structural basis of neuromuscular transmission: insights from Torpedo postsynaptic membranes. Q. Rev. Biophys. 2013, 46, 283-322.

11. Walsh, R. M., Jr.; Roh, S. H.; Gharpure, A.; Morales-Perez, C. L.; Teng, J.; Hibbs, R. E., Structural principles of distinct assemblies of the human alpha4beta2 nicotinic receptor. Nature 2018, 557, 261-265.

12. Morales-Perez, C. L.; Noviello, C. M.; Hibbs, R. E., X-ray structure of the human alpha 4 beta 2 nicotinic receptor. Nature 2016, 538, 411-415.

13. Nys, M.; Kesters, D.; Ulens, C., Structural insights into Cys-loop receptor function and ligand recognition. Biochem. Pharmacol. 2013, 86, 1042-1053.

14. Dajas-Bailador, F.; Wonnacott, S., Nicotinic acetylcholine receptors and the regulation of neuronal signalling. Trends Pharmacol. Sci. 2004, 25 (6), 317-324.

15. Shen, X. M.; Ohno, K. J.; Tsujino, A.; Brengman, J. M.; Gingold, M.; Sine, S. M.; Engel, A. G., Mutation causing severe myasthenia reveals functional asymmetry of AChR signature cystine loops in agonist binding and gating. J. Clin. Invest. 2003, 111, 497-505.

16. Kash, T. L.; Jenkins, A.; Kelley, J. C.; Trudell, J. R.; Harrison, N. L., Coupling of agonist binding to channel gating in the GABA(A) receptor. Nature 2003, 421, 272-275.

17. Bouzat, C.; Gumilar, F.; Spitzmaul, G.; Wang, H.; Rayes, D.; Hansen, S.; Taylor, P.; Sine, S., Coupling of agonist binding to channel gating in an ACh-binding protein linked to an ion channel. Nature 2004, 430 (7002), 896-900.

18. Chakrapani, S.; Bailey, T.; Auerbach, A., Gating dynamics of the acetylcholine receptor extracellular domain. J. Gen. Physiol. 2004, 123 (4), 341-356.

19. Xiu, X.; Hanek, A.; Wang, J.; Lester, H.; Dougherty, D., A unified view of the role of electrostatic interactions in modulating the gating of Cys loop receptors. J. Biol. Chem. 2005, 280 (50), 41655-41666.

20. Lee, W.; Sine, S., Principal pathway coupling agonist binding to channel gating in nicotinic receptors. Nature 2005, 438 (7065), 243-247. 
21. Grutter, T.; de Carvalho, L. P.; Dufresne, V.; Taly, A.; Edelstein, S. J.; Changeux, J. P., Molecular tuning of fast gating in pentameric ligand-gated ion channels. Proc. Natl. Acad. Sci. U. S. A. 2005, 102, 18207-18212.

22. Jha, A.; Cadugan, D. J.; Purohit, P.; Auerbach, A., Acetylcholine receptor gating at extracellular transmembrane domain interface: the cys-loop and M2-M3 linker. J. Gen. Physiol. 2007, 130, 547-558.

23. Bouzat, C.; Bartos, M.; Corradi, J.; Sine, S., The interface between extracellular and transmembrane domains of homomeric Cys-loop receptors governs open-channel lifetime and rate of desensitization. $J$. Neurosci. 2008, 28 (31), 7808-7819.

24. Lee, W.; Free, C.; Sine, S., Binding to Gating Transduction in Nicotinic Receptors: Cys-Loop Energetically Couples to Pre-M1 and M2-M3 Regions. J. Neurosci. 2009, 29 (10), 3189-3199.

25. Andersen, N.; Corradi, J.; Bartos, M.; Sine, S.; Bouzat, C., Functional Relationships between Agonist Binding Sites and Coupling Regions of Homomeric Cys-Loop Receptors. J. Neurosci. 2011, 31 (10), 3662-3669.

26. Campos-Caro, A.; Sala, S.; Ballesta, J. J.; Vicente-Agullo, F.; Criado, M.; Sala, F., A single residue in the M2-M3 loop is a major determinant of coupling between binding and gating in neuronal nicotinic receptors. Proc Natl Acad Sci U S A 1996, 93, 6118-23.

27. Lynch, J.; Rajendra, S.; Pierce, K.; Handford, C.; Barry, P.; Schofield, P., Identification of intracellular and extracellular domains mediating signal transduction in the inhibitory glycine receptor chloride channel. EMBO J. 1997, 16 (1), 110-120.

28. Rovira, J. C.; Ballesta, J. J.; Vicente-Agullo, F.; Campos-Caro, A.; Criado, M.; Sala, F.; Sala, S., A residue in the middle of the M2-M3 loop of the beta4 subunit specifically affects gating of neuronal nicotinic receptors. FEBS Lett. 1998, 433, 89-92.

29. Rovira, J. C.; Vicente-Agullo, F.; Campos-Caro, A.; Criado, M.; Sala, F.; Sala, S.; Ballesta, J. J., Gating of alpha(3)beta(4) neuronal nicotinic receptor can be controlled by the loop M2-M3 of both alpha(3) and beta(4) subunits. Pflugers Archiv. 1999, 439, 86-92.

30. Grosman, C.; Salamone, F. N.; Sine, S. M.; Auerbach, A., The extracellular linker of muscle acetylcholine receptor channels is a gating control element. J. Gen. Physiol. 2000, 116, 327-340.

31. Huggins, D. J.; Biggin, P. C.; Dämgen, M. A.; Essex, J. W.; Harris, S. A.; Henchman, R. H.; Khalid, S.; Kuzmanic, A.; Laughton, C. A.; Michel, J.; Mulholland, A. J.; Rosta, E.; Sansom, M. S. P.; van der Kamp, M. W., Biomolecular simulations: From dynamics and mechanisms to computational assays of biological activity. WIRES Comput. Mol. Sci. 2018, e1393, 1-23.

32. Oliveira, A. S. F.; Shoemark, D. K.; Campello, H. R.; Gallagher, T.; Sessions, R. B.; Mulholland, A. J., Identification of the initial steps in signal transduction in the $\alpha 4 \beta 2$ nicotinic receptor: insights from equilibrium and nonequilibrium simulations. Structure 2019, 27, 1171-1183.

33. Jadey, S.; Auerbach, A., An integrated catch-and-hold mechanism activates nicotinic acetylcholine receptors. J. Gen. Physiol. 2012, 140, 17-28.

34. Purohit, P.; Auerbach, A., Loop C and the mechanism of acetylcholine receptor-channel gating. J. Gen. Physiol. 2013, 141, 467-478.

35. Pless, S. A.; Sivilotti, L. G., A tale of ligands big and small: An update on how pentameric ligand-gated ion channels interact with agonists and proteins. Curr. Opin. Physiol. 2018, 2, 19-26.

36. Van Arnam, E. B.; Dougherty, D. A., Functional probes of drug-receptor interactions implicated by structural studies: Cys-loop receptors provide a fertile testing ground. J. Med. Chem. 2014, 57, 6289-6300.

37. Newell, J. G.; Czajkowski, C., The GABA(A) receptor alpha(1) subunit Pro(174)-Asp(191) segment is involved in GABA binding and channel gating. J. Biol. Chem. 2003, 278, 13166-13172.

38. Thompson, A. J.; Padgett, C. L.; Lummis, S. C. R., Mutagenesis and molecular modeling reveal the importance of the 5-HT3 receptor F-loop. J. Biol. Chem. 2006, 281, 16576-16582.

39. Bafna, P. A.; Purohit, P. G.; Auerbach, A., Gating at the mouth of the acetylcholine receptor channel: energetic consequences of mutations in the alphaM2-cap. PLoS One 2008, 3, e2515.

40. Lee, W. Y.; Free, C. R.; Sine, S. M., Nicotinic receptor interloop proline anchors beta1-beta2 and Cys loops in coupling agonist binding to channel gating. J. Gen. Physiol. 2008, 132, 265-78.

41. Kusama, T.; Wang, J.; Spivak, C.; Uhl, G., Mutagenesis of the GABA RHO-1 receptor alters agonist affinity and channel gating. Neuroreport 1994, 5 (10), 1209-1212. 
42. Lewis, T.; Sivilotti, L.; Colquhoun, D.; Gardiner, R.; Schoepfer, R.; Rees, M., Properties of human glycine receptors containing the hyperekplexia mutation alpha 1(K276E) expressed in Xenopus oocytes. J. Physiol.-London 1998, 507 (1), 25-40.

43. O'Shea, S.; Harrison, N., Arg-274 and Leu-277 of the gamma-aminobutyric acid type A receptor alpha(2) subunit define agonist efficacy and potency. J. Biol. Chem. 2000, 275 (30), 2276422768.

44. Sigel, E.; Buhr, A.; Baur, R., Role of the conserved lysine residue in the middle of the predicted extracellular loop between M2 and M3 in the GABA(A) receptor. J. Neurochem. 1999, 73 (4), 1758-1764.

45. Deane, C.; Lummis, S., The role and predicted propensity of conserved proline residues in the 5-HT3 receptor. J. Biol. Chem. 2001, 276 (41), 37962-37966.

46. Jensen, M. O.; Park, S.; Tajkhorshid, E.; Schulten, K., Energetics of glycerol conduction through aquaglyceroporin GlpF. Proc. Natl. Acad. Sci. U. S. A. 2002, 99, 6731-6736.

47. De Fabritiis, G.; Coveney, P. V.; Villa-Freixa, J., Energetics of K+ permeability through Gramicidin A by forward-reverse steered molecular dynamics. Proteins 2008, 73, 185-194.

48. Ngo, V.; Stefanovski, D.; Haas, S.; Farley, R. A., Non-equilibrium dynamics contribute to ion selectivity in the KcsA channel. PLoS One 2014, 9, e86079.

49. Ngo, V.; Wang, Y.; Haas, S.; Noskov, S. Y.; Farley, R. A., K+ Block Is the Mechanism of Functional Asymmetry in Bacterial Na(v) Channels. PLoS Comput. Biol. 2016, 12, e1004482.

50. Ciccotti, G.; Jacucci, G.; McDonald, I. R., Thought-Experiments by Molecular Dynamics. J. Stat. Phys. 1979, 21, 1-21.

51. Ciccotti, G., Molecular dynamics simulation of non equilibrium phenomena and rare dynamical events. In Computer simulation in material science, Meyer M, P. V., Ed. Kluwer Academic Publishers: 1991; pp 119-137.

52. Ciccotti, G.; Ferrario, M., Non-equilibrium by molecular dynamics: a dynamical approach. Mol. Simul. 2016, 42 (16), 1385-1400.

53. Bouzat, C.; Mukhtasimova, N., The nicotinic acetylcholine receptor as a molecular machine for neuromuscular transmission. Curr. Opin. Physiol. 2018, 04, 40-48.

54. Corringer, P. J.; Poitevin, F.; Prevost, M. S.; Sauguet, L.; Delarue, M.; Changeux, J. P., Structure and pharmacology of pentameric receptor channels: from bacteria to brain. Structure 2012, 20, 941-956.

55. Newell, J. G.; McDevitt, R. A.; Czajkowski, C., Mutation of glutamate 155 of the GABA(A) receptor beta(2) subunit produces a spontaneously open channel: A trigger for channel activation. $J$. Neurosci. 2004, 24, 11226-11235.

56. Padgett, C. L.; Lummis, S. C. R., The F-loop of the GABA(A) receptor gamma(2) subunit contributes to benzodiazepine modulation. J. Biol. Chem. 2008, 283, 2702-2708.

57. Pless, S. A.; Lynch, J. W., Ligand-specific Conformational Changes in the alpha 1 Glycine Receptor Ligand-binding Domain. J. Biol. Chem. 2009, 284, 15847-15856.

58. Khatri, A.; Sedelnikova, A.; Weiss, D. S., Structural Rearrangements in Loop F of the GABA Receptor Signal Ligand Binding, Not Channel Activation. Biophys. J. 2009, 96, 45-55.

59. Eisele, J. L.; Bertrand, S.; Galzi, J. L.; Devillers-Thiery, A.; Changeux, J. P.; Bertrand, D., Chimaeric nicotinic-serotonergic receptor combines distinct ligand binding and channel specificities.

Nature 1993, 366, 479-483.

60. Magnus, C. J.; Lee, P. H.; Atasoy, D.; Su, H. H.; Looger, L. L.; Sternson, S. M., Chemical and genetic engineering of selective ion channel-ligand interactions. Science 2011, 333, 1292-1296. 
TOC graphic

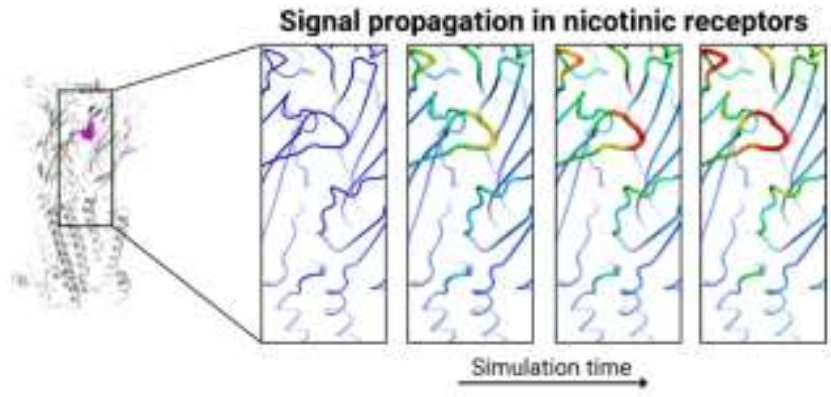




\section{A general mechanism for signal propagation in the nicotinic acetylcholine receptor family}

Ana Sofia F. Oliveira, Christopher J. Edsall, Christopher J. Woods, Phil Bates, Gerardo Viedma Nunez, Susan Wonnacott, Isabel Bermudez, Giovanni Ciccotti, Timothy C. Gallagher, Richard B. Sessions and Adrian J. Mulholland

\section{SUPPORTING INFORMATION}

\section{Starting Structure}

A homology model for the extracellular and transmembrane domains of the human $\alpha 7$ nicotinic receptor was constructed using the $\alpha 4$ subunit from the crystal structure of the human $\alpha 4 \beta 2$ nicotinic receptor (PDB code: $5 \mathrm{KXI})^{1}$ as a template. This X-ray structure is thought to reflect a desensitised state of the receptor with two agonists molecules present in the binding pockets and a closed, non-conducting ion channel ${ }^{1}$.

The sequence for the human $\alpha 7$ subunit was obtained from the UniProt database ${ }^{2}$ (code P36544) and aligned with the template using Clustal Omega ${ }^{3,4}$. The sequence identity between the $\alpha 7$ and the $\alpha 4$ subunits is $48 \%$, and it was determined using BLAST ${ }^{5}$. It should be noted that in this work, the intracellular region of the receptor (residues 345354) was not modelled due to the lack of structural information. Twenty different models were generated with the MODELLER 9v17 ${ }^{6}$, and the one with the lowest value for MODELLER's objective function was selected and further validated using PROCHECK $^{7}$. Nicotine was modelled in two of the five binding pockets with binding modes similar to nicotine in the structure of the $\alpha 4 \beta 2$ receptor $^{1}$. It has been shown experimentally that the binding of agonists to two nonconsecutive binding pockets enables proper activation of homomeric Cys-loop receptors. ${ }^{8}$ Unless stated otherwise, all residues are numbered according to the human $\alpha 7$ sequence (www.uniprot.org/uniprot/P36544). 
$\alpha 2$
$\alpha 3$
$\alpha 4$
$\alpha 5$
$\alpha 6$
$\alpha 7$
$\alpha 9$
$\alpha 1$
$\beta 2$
$\beta 4$
8
$\varepsilon$

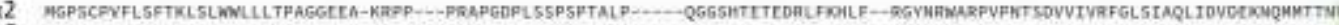
D.

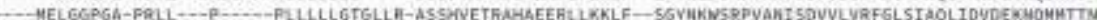

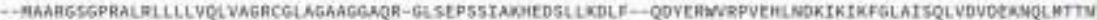

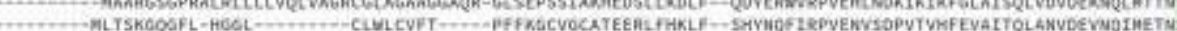

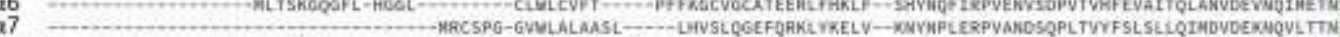

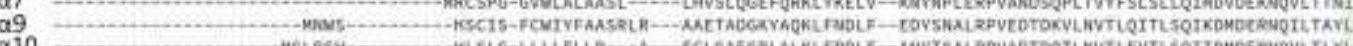

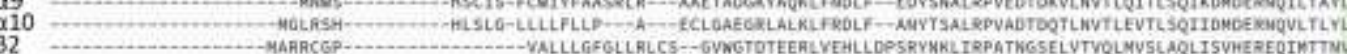

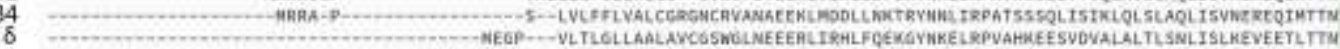

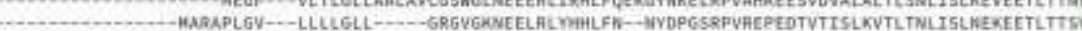
$\operatorname{Logp} 0$
Loop A
$\operatorname{Lacop} E$
Cys loop Loop $B$

$\alpha 2$ whǫews

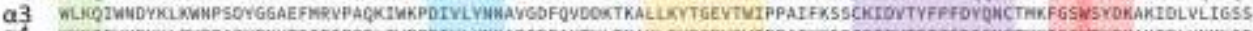

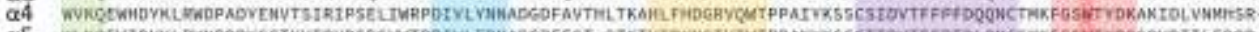

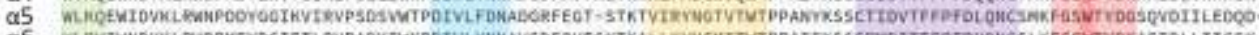

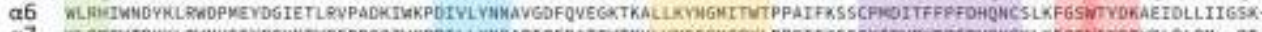

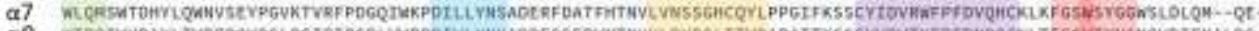

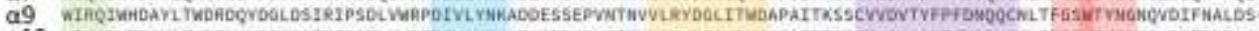

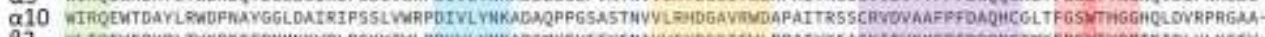

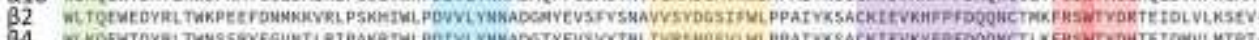

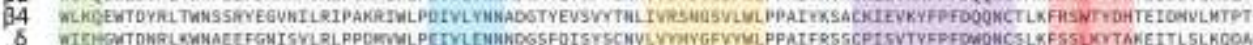

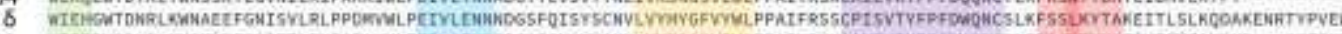
ETotowQ
Loop F
Loop C
M2-M3 linker

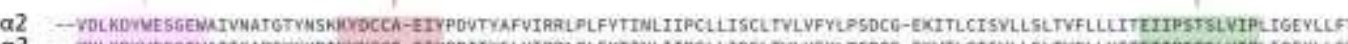

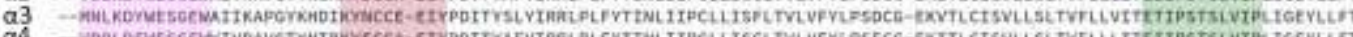

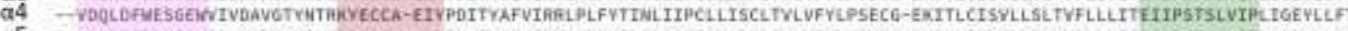

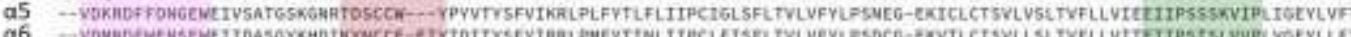

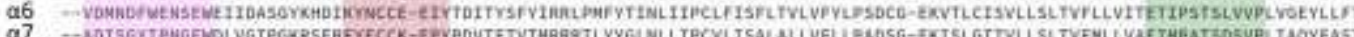

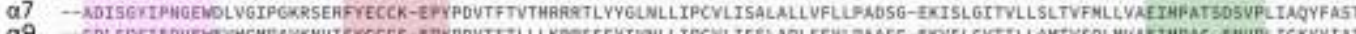

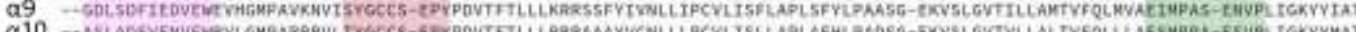

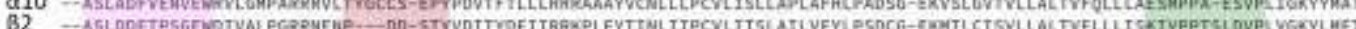

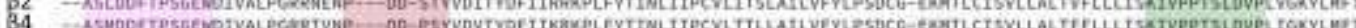
סै

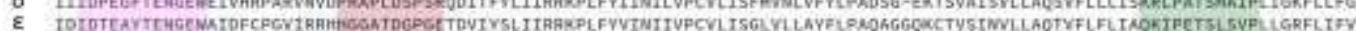

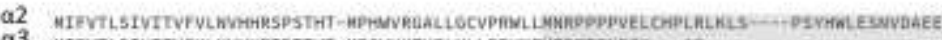

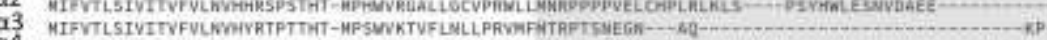

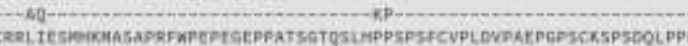

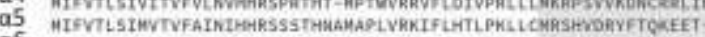

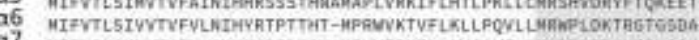

d7 MIIVSLSVYVTVIVL Q

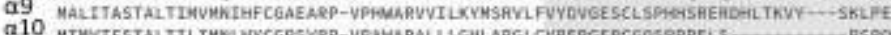

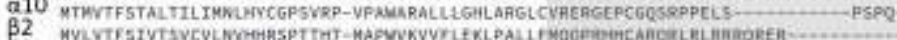

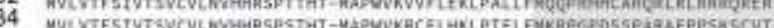

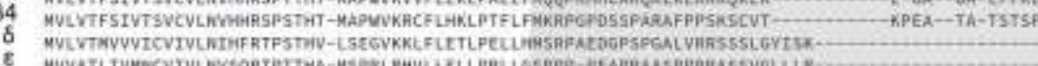

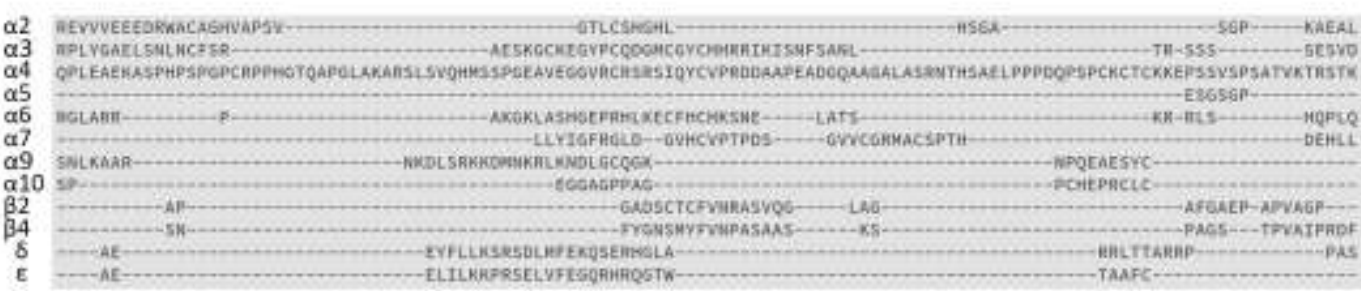

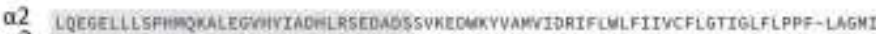

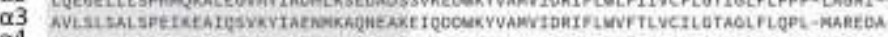

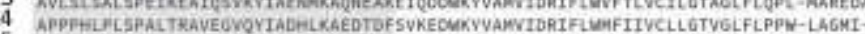

a5 5 -

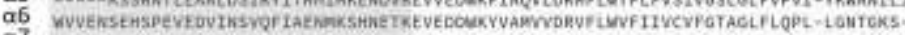

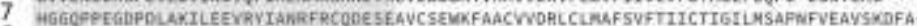

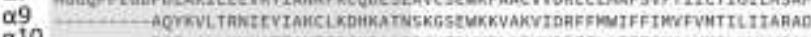

-

WESsce

Figure S1. Sequence alignments for several nAChR subunits. All sequences were taken from the UniProt database. 2, namely codes Q15822, P32297, P43681, P30532, Q15825, P36544, Q9UGM1, Q9GZZ6, P17787, P30926, Q07001 and Q04844 for the $\alpha 2, \alpha 3, \alpha 4, \alpha 5, \alpha 6, \alpha 7, \alpha 9, \alpha 10, \beta 2, \beta 4, \delta$ and $\varepsilon$ subunits, 
respectively. The loops forming the binding pockets and other structural elements are labelled accordingly. The intracellular regions are highlighted in grey. The aromatic residue in loop B known to directly interact with the ammonium group of the agonist ${ }^{9}$ is coloured in dark red. Please zoom into the image for detailed visualisation of the sequence alignments.
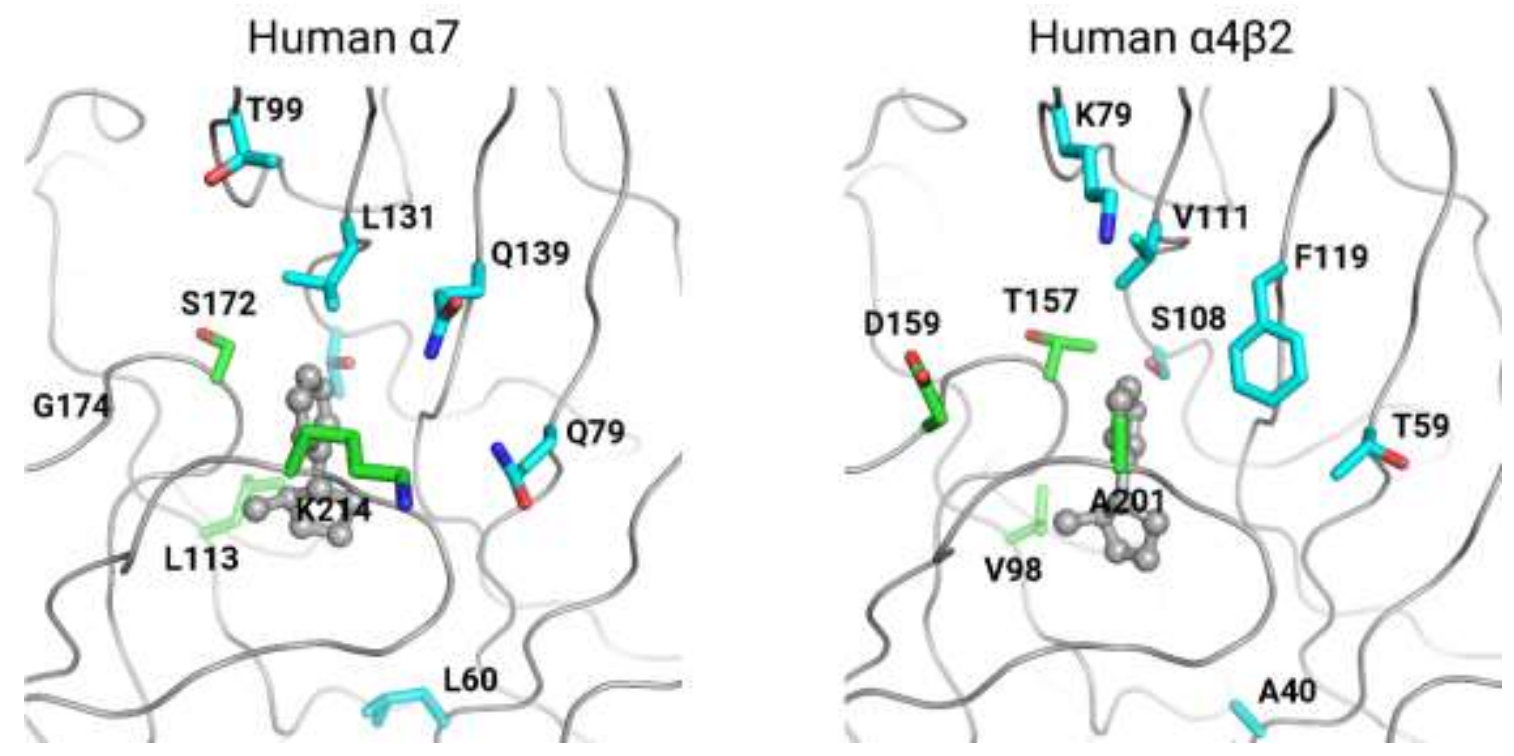

Figure S2. Differences in the binding pocket between the human $\alpha 7$ and the $\alpha 4 \beta 2$ subtypes. For clarity, the residues numbering in the $\alpha 7$ subtype refers to the sequence (UniProt ${ }^{2}$ code P36544) whereas in the $\alpha 4 \beta 2$ subtype it relates to the 5KXI X-ray structure. ${ }^{1}$ The residues that are different between the two subtypes are shown with green (principal subunit) and cyan (complementary subunit) sticks. Nicotine is represented in balls-and-sticks. Note that the structure representing the $\alpha 7$ subtype (left side image) is the homology model constructed using crystal structure of the $\alpha 4 \beta 2$ subtype $^{1}$ as template. The structure for the $\alpha 4 \beta 2$ subtype (right side image) corresponds to the crystal structure (PDB code: $5 \mathrm{KXI}){ }^{1}$

\section{Protonation state of protonatable residues}

The protonation state of each titrable site at $\mathrm{pH} 7.0$ was determined using a combination of Poisson-Boltzmann calculations and Metropolis Monte Carlo simulations similarly to $^{10}$. The Poisson-Boltzmann calculations were performed using MEAD (version 2.2.9) ${ }^{11-13}$, and the Metropolis Monte Carlo simulations were done with PETIT (version 1.6). ${ }^{14}$ All the settings were similar to the ones described $i^{10}$. Based on these calculations, all lysines, arginines, aspartates, and glutamates were considered charged. Histidines were found to be in different protonation states: H85, H127, H137, H318 and H320 were considered neutral whereas H163 and H319 were (positively) charged. 
The nicotine molecules were treated as positively charged because the $\mathrm{pKa}$ value for its pyrrolidine $\mathrm{N}$ site is $\sim 8 .{ }^{15}$ The overall charge for the receptor-nicotine complex was -23 .

\section{Equilibrium simulations}

The receptor was inserted in a pre-equilibrated POPC bilayer using LAMBADA and InflateGRO $2^{16}$, resulting in a membrane-protein system containing 420 lipids. Two systems were prepared, one with no agonist bound to the receptor (hereafter named APO) and a second one, with nicotine bound in two of the five binding pockets (hereafter called NCT). All systems were solvated using TIP3P water molecules ${ }^{17}$, and an ionic concentration of $0.1 \mathrm{M}$ sodium chloride was used.

All equilibrium MD simulations were performed using GROMACS (version 5.1.4) ${ }^{18}$ on the University of Bristol's High-Performance Computer, BlueCrystal (Phase 4). The Amber ff99SB-ILDN ${ }^{19}$ force-field was used to describe the protein, whereas the Slipids forcefield was used for the membrane. ${ }^{20,21}$ The parameters for the protonated nicotine were taken from our previously published work ${ }^{10,22}$, in which (as here) the $\mathrm{R}$ configuration was chosen for the nitrogen stereocenter, as observed in the human $\alpha 4 \beta 2$ crystal structure. ${ }^{1}$ All simulations were performed at a constant temperature of $310 \mathrm{~K}$, and the velocity-rescaling thermostat ${ }^{23}$ was used, with separate couplings for the solutes and solvent, using a relaxation time constant of 0.1 ps. A Berendsen barostat ${ }^{24}$ was used to keep the pressure at 1 bar, with a coupling constant of $1.0 \mathrm{ps}$ and isothermal compressibility of $4.5 \times 10^{-5} \mathrm{bar}^{-1}$. The pressure was coupled semi-isotropically, resulting in the independent coupling of the lateral $\mathrm{P}(\mathrm{x}+\mathrm{y})$ and perpendicular $(\mathrm{Pz})$ pressures. A time step of 2 fs was used for integrating the equations of motion. Nonbonded long-range electrostatic interactions were calculated using $\mathrm{PME}^{25}$ beyond a 12 $\AA$ cutoff. The same $12 \AA$ cutoff was used for the van der Waals interactions with longrange dispersion corrections for the energy and pressure. ${ }^{26}$ The neighbours list was updated every 20 steps. All bonds were constrained to their equilibrium lengths with the LINCS algorithm ${ }^{27}$ except for the water molecules, which were kept rigid with the SETTLE algorithm. ${ }^{28}$

The solvated protein-membrane systems were energy minimised, equilibrated (for 20 ns) and simulated according to the protocol described previously in ${ }^{10}$. Ten unrestrained 
MD simulations, each $500 \mathrm{~ns}$ long, were performed for each system, totalling $10 \mu \mathrm{s}$ of simulation.

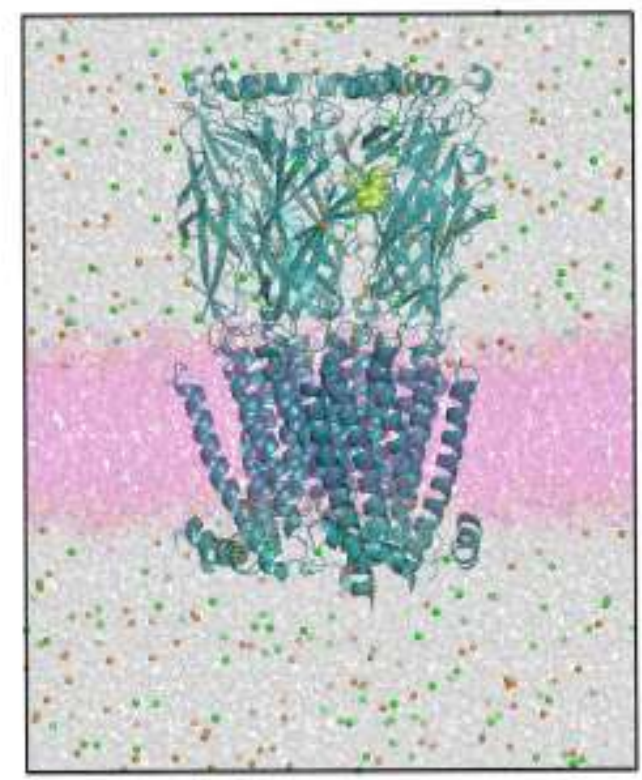

Figure S3. View of the nicotine-bound $\alpha 7$ system. The protein is rendered as a cartoon, whereas the POPC lipids are represented as magenta sticks. Nicotine (yellow), $\mathrm{Na}^{+}$(orange) and $\mathrm{Cl}^{-}$(green) ions are shown with spheres.

It should be noted, that similarly to the X-ray structure ${ }^{1}$ used as a template for our model, our $\alpha 7$ receptor model is thought to represent a desensitised state in which the ion channel adopts a closed V-shaped conformation despite the agonists bound in the binding pockets. According to the MWC allosteric model, a minimum of four conformational states (Figure S4) are needed to represent the working cycle of pentameric ligand-gated ion channels $(\mathrm{pLGICs})^{29}$ : three primary states (namely Resting, Active, and Desensitised) and an intermediate state (which may include multiple intermediate conformations). According to this model, the binding of agonists to the binding pockets induces the transition from a closed resting to an open, active conformation. Prolonged binding of the agonist leads to a high affinity, agonist-bound, nonconducting desensitised state. ${ }^{29}$ The exit of the agonist from the binding pockets returns the receptor to the resting state again. ${ }^{29}$ The equilibrium simulations reported here focus on Desensitised (ligand bound, NCT) state, while the APO simulations give an indication of the Desensitised to Resting transition 
(red arrow in Figure S4). We do not aim to model channel opening, and these simulations do not do so.

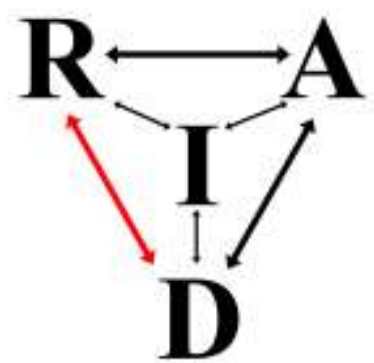

Figure S4. Simplified conformational state scheme representation for the working cycle of pLGICs. R, A, D and I refer to resting, active, desensitised and intermediate states, respectively (see text for more details). The red arrow corresponds to the state transition sampled during our equilibrium simulations, which begin in what is thought to be the desensitized state.

\section{Nonequilibrium simulations}

To study signal propagation between the binding pockets and the TMDs, a large set (450) of very short (5 ns) nonequilibrium simulations was performed. These simulations drive, and allow for the characterisation of rapid conformational changes in the system by using the Kubo-Onsager approach ${ }^{30-32}$. It should be noted that the subtraction technique $^{30-32}$ is just a particular instance of the Kubo-Onsager approach that can be used when the two sets of simulations (with and without a perturbation) are correlated ${ }^{30}$, and it allows for the cancellation of the noise arising from the intrinsic fluctuations of the system and for the identification of the response to the perturbation in a statistically significant way. Note that in the long equilibrium simulations, due to the limited sampling (10 replicates/system) and the lack of a driving force, it is not possible to determine the order of the events associated with any propagation of conformational changes from the binding pockets to the TMDs.

The starting conformations for the short nonequilibrium simulations were extracted from the equilibrated part of the $500 \mathrm{~ns}$ equilibrium NCT simulations (from 50-500 ns). Conformations were taken every 10 ns (45 frames per replicate), and in each, the nicotine molecules were removed from the binding pockets (Figure S5), i.e. simply annihilated. Note that in order to maintain the electroneutrality of the system required 
for PME, two negative ions were also removed from the solvent. The effect of nicotine annihilation was studied by the Kubo-Onsager method. ${ }^{30-32}$ According to this method ${ }^{30-}$ ${ }^{32}$, the response (over the first few nanoseconds) of a system to a perturbation can be directly measured by averaging a given property (in this case, the position of the $\mathrm{C}_{\alpha}$ atoms) in perturbed (APO) and unperturbed simulations (NCT) at a given time, as long as enough data is gathered (Figure S5A). For each pair of unperturbed equilibrium NCT and perturbed nonequilibrium APO simulations, the difference in position for each $\mathrm{C} \alpha$ atoms was determined at equivalent points in time, namely after 0, 50, 500, 1000, 3000 and 5000 ps of simulation (see Figure S5B). The use of the $\mathrm{C} \alpha$ atoms is this analysis is a straightforward way to identify the most pronounced conformational rearrangements while reducing the noise coming from the fluctuations of the side-chains. Note that as we are only calculating the difference in position between 2 conformations, no special code is needed and, any tool able to calculate the distance between two atoms (e.g. rms or distance tools in GROMACS ${ }^{18}$ ) can, in principle, be used for this analysis. The positional deviations values obtained at each point in time were then average over all 450 simulations. Since the data is collected from a large number of simulations (450), we can be sure of the statistical significance of the structural changes identified due to the low standard error on the averages.

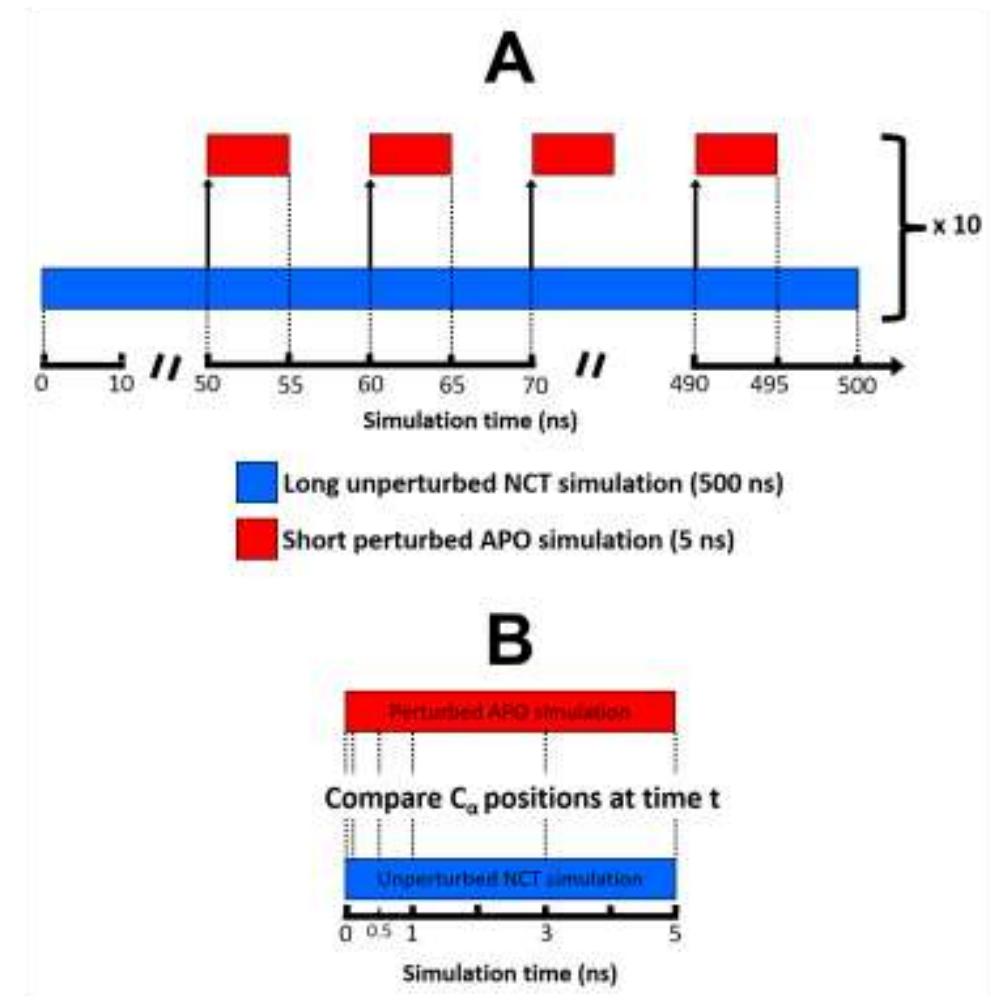


Figure S5. Scheme for the nonequilibrium simulations. A. From the model of the human $\alpha 7$ nicotinic receptor structure with nicotine bound, 10 equilibrium MD simulations, $500 \mathrm{~ns}$ each, were performed (blue). These simulations (NCT bound, shonw in blue) were used to generate starting structures for the short APO nonequilibrium simulations (red): conformations were sampled every $10 \mathrm{~ns}$, beginning from the $50 \mathrm{~ns}$ structures of the NCT simulations, giving a total of 450 short APO simulations. B. For each pair of unperturbed NCT and perturbed APO simulations, the positional deviations of each $\mathrm{C} \alpha$ at equivalent times (namely 0, 50, 500, 1000, 3000 and 5000 ps) were determined.

It is important to note that the removal of the ligands from the binding pockets is not intended to represent the physical process of unbinding. Its primary purpose is to create a perturbation in the system and force a response from the receptor. Furthermore, it also should be noted that these nonequilibrium simulations (due to their short timescales and the artificial nature of the perturbation) are not attempting to explain the entire mechanism of gating or how ligand binding and unbinding induce channel opening and closing. Instead, they allow the identification of the first conformational changes associated with signal propagation. Nonetheless, the structural elements of the communication pathway revealed by the nonequilibrium simulations are likely to be involved in the response to binding and unbinding of agonists and in the conformational rearrangements occurring in the transitions between states. The simulation conditions for the nonequilibrium simulations were identical to those described above for the equilibrium ones.

Having used the University of Bristol HPC 'BlueCrystal' for the equilibrium simulations, in this part of the work (nonequilibrium MD), we made use of public cloud computing rather than our institution's HPC. Specifically, we made use of the Oracle Cloud Infrastructure (ttps://cloud.oracle.com/en_US/iaas) and the bare-metal instances it provides. The basic design mirrors an on-premises HPC cluster: there is a head node (in this case a virtual machine of "shape" VM.Standard2.2) and a number of compute nodes (one hundred instances of the bare-metal node BM.Standard2.52). All the nodes have access to a shared filesystem using the NFS protocol. The expected I/O requirements for one hundred simultaneous GROMACS jobs didn't require a more sophisticated shared filesystem or an alternative like an object store. The compute nodes are dual-socket servers with two "Intel Xeon Platinum 8167M CPU @ 2.00GHz" CPUs giving 52 cores and 104 hyperthreads per node and $768 \mathrm{GiB}$ of RAM with a two 10 
$\mathrm{Gb} / \mathrm{s}$ Ethernet network interfaces. These are the Oracle Server X7-2 model (www.oracle.com/us/products/servers/arch-wp-oracle-servers-x7-2-x7-21-3852567.pdf) servers. The resources were located in the us-ashburn-1 region in two availability domains. To configure the cluster, two tools were used with a separation of concerns, each managing a different layer of the software stack. Terraform (https://www.terraform.io) was used to manage the deployment and configuration of the cloud resources: the instances, the virtual cloud networks and storage up to the point of installing the operating system. Ansible (https://www.ansible.com) was used to manage the configuration of the operating systems, installing the job scheduler and cluster configuration and installing the application. With this approach, simply changing the Terraform 'provider' would enable the same system to be built in another public or private cloud. All of this was managed via cluster-in-the-cloud (https://doi.org/10.5281/zenodo.3246253). The operating system for all nodes was Oracle-Linux-7.502018.06.14-0 based on RedHat Enterprise Linux 7.x. The job scheduler used for the cluster was SLURM version 17.11.7. GROMACS itself was built from source (www.gromacs.org) in to an operating system native package (RPM) format using the .SPEC file in the Ansible repository. All simulations were completed over a real-time period of 5 days. Benchmarking against BlueCrystal Phase 4 indicated that the simulations would have taken $\sim 90$ days to run locally. The speed-up was a combination of GROMACS running faster on the cloud HPC compute nodes, and the elastic scaling nature of the cloud providing more on-demand compute capacity versus what was available locally on a shared multi-user cluster. Each individual GROMACS simulation took $\sim 8$ hours to complete on a cloud $2 \times 28$ core Platinum Xeon $8167 \mathrm{M}$ node versus $\sim 17$ hours on a BlueCrystal Phase 4 2x14 core Gold Xeon E5-2680v4 node. BlueCrystal Phase 4 is a shared cluster with many users, and we estimated that only 10 nodes would be available on average on this shared resource via the queue. This is compared to 100 nodes that were available elastically on the cloud.

\section{Analysis}

All the analyses were performed using GROMACS ${ }^{18}$ tools and in-house tools. All molecular images were created with PyMOL. ${ }^{33,34}$ PCA analysis was used to examine 
the sampling and equilibration of the replicates (similarly to, e.g. ${ }^{35-37}$ ) and to identify the most relevant motions in the protein. All replicates for each system were combined before the analysis so that they all share the same subspace and could be directly compared. Each PCA trajectory contained one conformation per nanosecond per replicate (totaling 5001 and 2251 frames for the equilibrium and nonequilibrium simulations, respectively). The two principal components (PC1 and PC2) were used to assess the equilibration/relaxation of the simulations, and all systems were considered equilibrated after 50 ns. The Kubo-Onsager approach ${ }^{30-32}$ (see above) was used to analyse the nonequilibrium simulations and determine the response of the system to the annihilation of nicotine.

\section{Conformational stability of the $\alpha 7 \mathrm{nAChR}$}

The structural stability of the receptor in the APO and NCT systems was examined by monitoring two system properties, namely the $\mathrm{C}_{\alpha}$ root mean square deviation (RMSD) from the starting structure and the secondary structure content. As can be seen in Figure S6, both systems remain stable over the $500 \mathrm{~ns}$ simulations, and the average $\mathrm{C}_{\alpha}$ RMSD profiles show a plateau after $50 \mathrm{~ns}$. The average RMSD profiles slowly increased during the first tens of nanoseconds of simulation, reaching global values of around $0.37 \mathrm{~nm}$ for both systems. The stability of the systems is further demonstrated by the analysis of the secondary structure content of the receptor (using $\operatorname{DSSP}^{38}$ ) with only a small secondary structure loss $(<2 \%)$.

Principal component analysis (PCA) was used to check the relaxation and sampling of the replicates. ${ }^{35-37}$ This analysis clearly shows (see Figure S6C) that the different replicates sample different regions of conformational space, improving the overall sampling for each system, thus mitigating the sampling problem. 

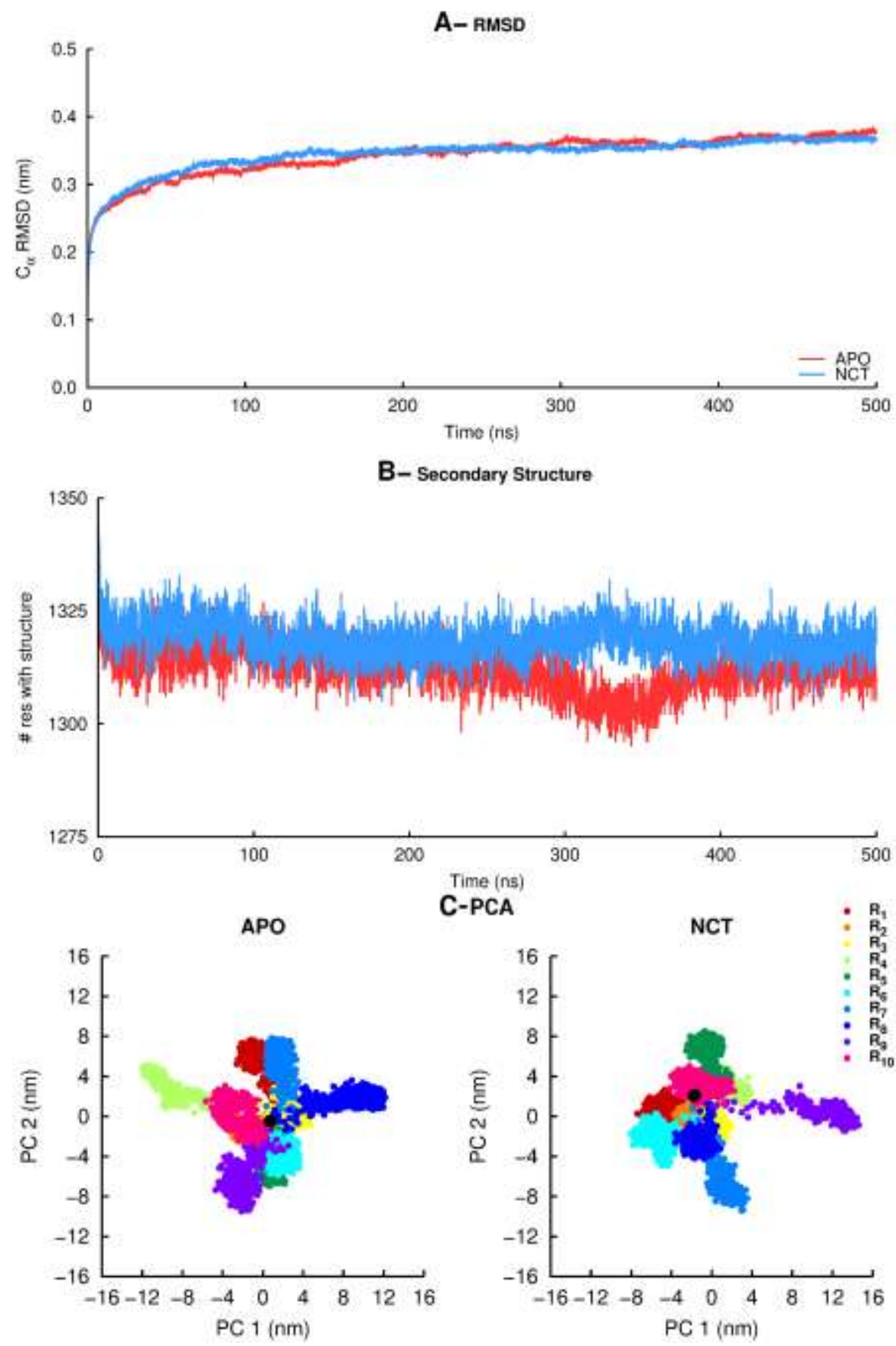

Figure S6. Equilibration and stability of the APO and NCT systems. A. Temporal evolution of the average $\mathrm{C}_{\alpha}$ RMSD for the APO (red) and NCT (blue) systems. The $\mathrm{C}_{\alpha}$ RMSD was calculated relative to the starting structures, and the averages were obtained over all replicates. B. Number of residues with secondary structure for the APO (red) and NCT (blue) systems. The DSSP software ${ }^{38}$ was used for the assignment of the secondary structure. C. PCA analysis of all replicates for the APO and NCT systems. All 10 replicates for each system were combined before the analysis and each PCA trajectory contained one conformation per nanosecond per replicate (totaling 5001 frames) with all the $\mathrm{C}_{\alpha}$ atoms of the protein. The black dot corresponds to the structure used as the starting point for all the replicates. For a detailed view, please zoom into the image. 


\section{Dynamic behaviour of the nicotine ligands}

The dynamic behaviour of nicotine was monitored over the $10 \mu$ s of simulation time and, despite some fluctuations, both nicotine molecules maintained their positions throughout (Figure S7). Nevertheless, and despite the mobility of the ligands, the canonical interaction between the positively charged nitrogen atom of nicotine and $\operatorname{TrpB}$ (W171 from the principal subunits) ${ }^{39-41}$ is mostly present in both binding pockets (see Figures S8). This interaction has been shown experimentally to provide an anchor point for the agonist in the binding site. ${ }^{39}$

The analysis of the dihedral angle between the pyridine and pyrrolidine rings of nicotine showed two possible binding modes (Figure S9): a preferred one (around $115^{\circ}$ ) observed in $68 \%$ of cases and a second (around $-65^{\circ}$ ) found for the remaining $32 \%$. 
A

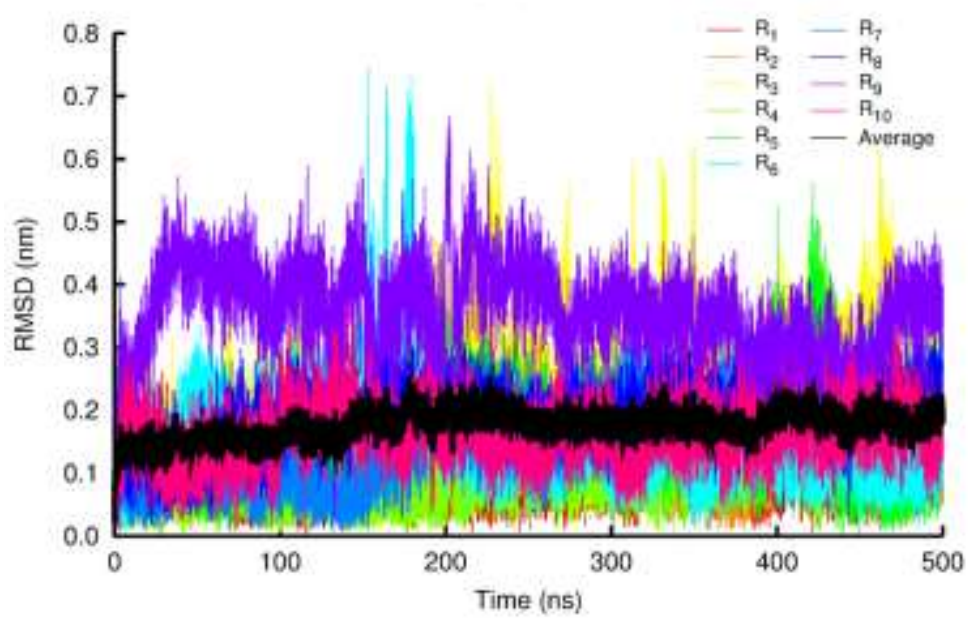

C

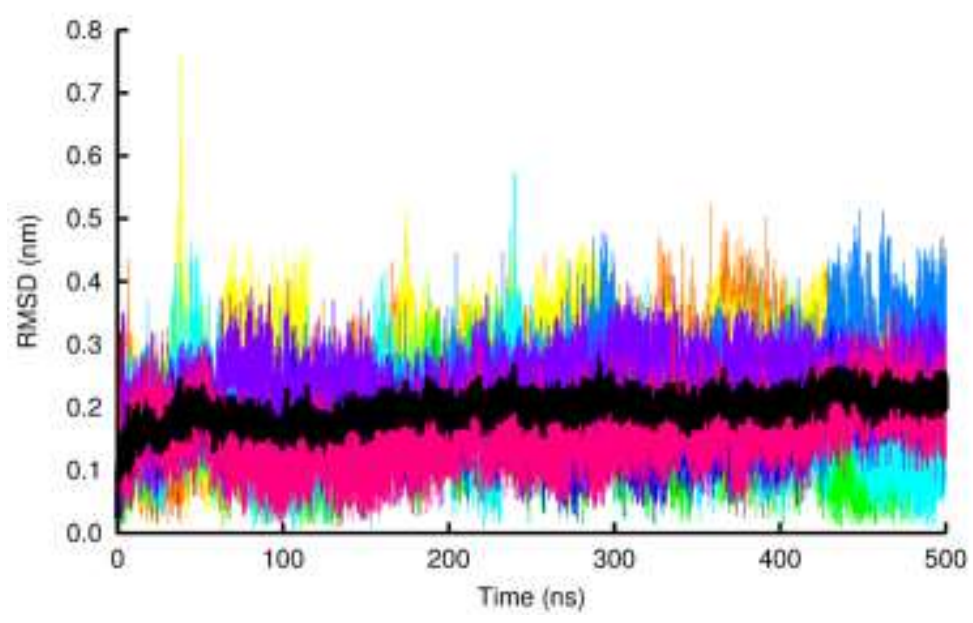

B

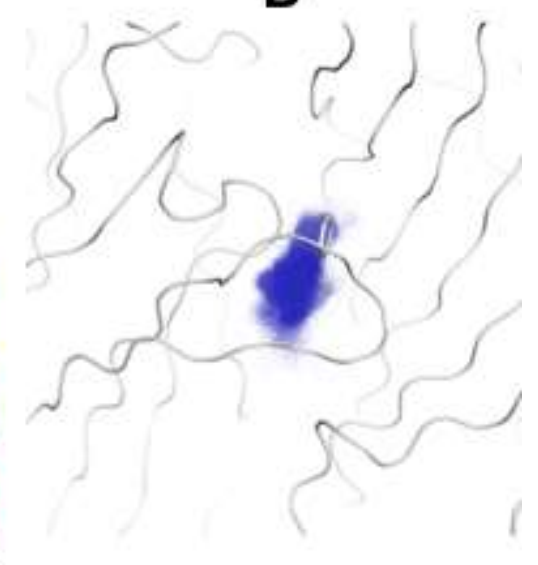

D

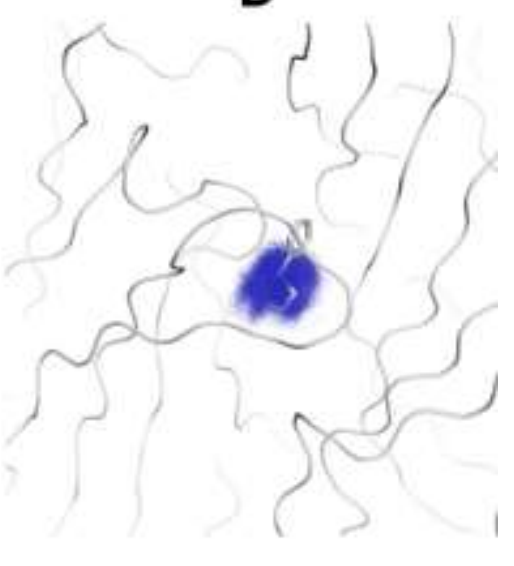

Figure S7. Time evolution of the position of nicotine (NCT) during the equilibrium MD simulations.

A, C. RMSD of the protonated secondary amine $\mathrm{N}$ of the nicotine molecules located in the first binding (A) and second (C) binding pocket. The RMSD was calculated relative to nicotine's initial position. The averages represented (black line) were obtained over all 10 replicates. B, D. Superposition of the positions adopted by the protonated secondary amine $\mathrm{N}$ atom of nicotine in the first (B) and second (D) binding pockets during the MD simulations. In this image, each small blue dot represents the position of nicotine's protonated secondary amine $\mathrm{N}$ atom. The structure used as the starting point for the simulations is shown in gray and, for clarity, the transmembrane domains are omitted. 


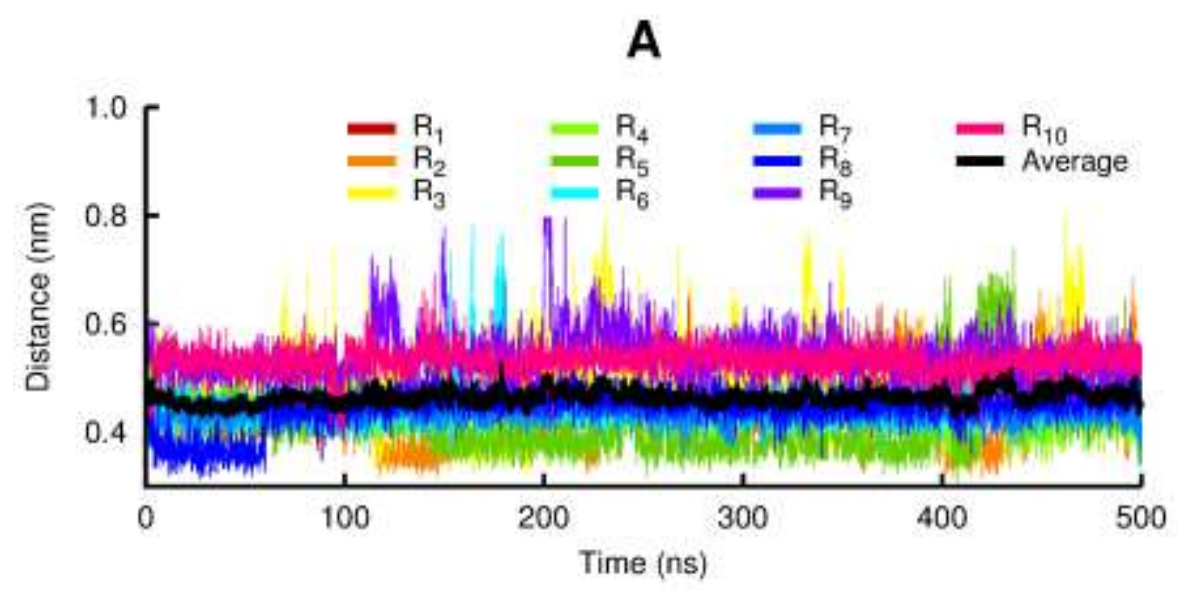

B

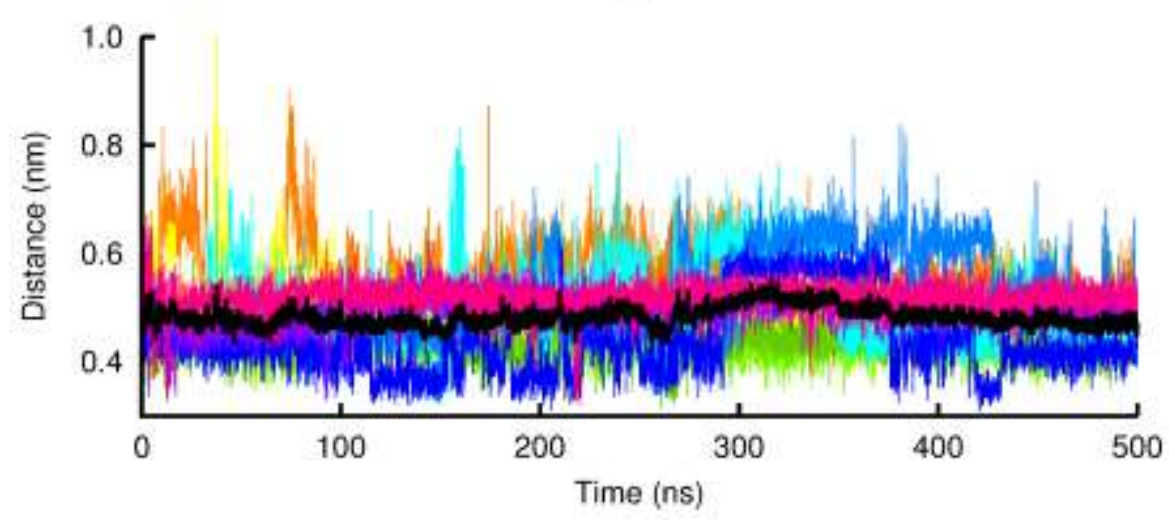

C

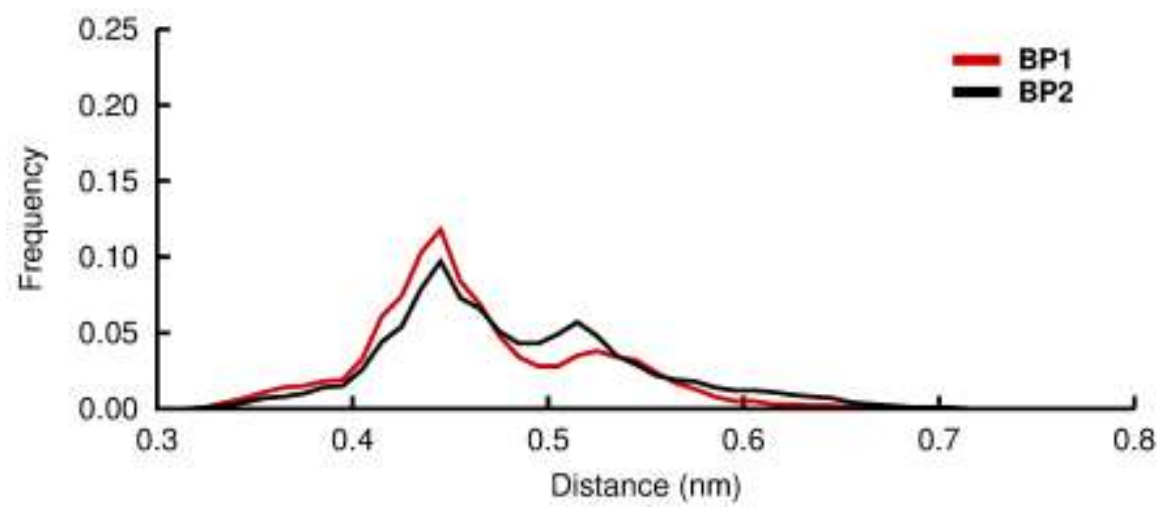

Figure S8. TrpB-nicotine distance over the simulation time. Distance between the side chain of $\operatorname{TrpB}$ (W171 in the principal subunits) and the charged secondary amine $\mathrm{N}$ atom of nicotine for the first (A) and second (B) binding pockets. C. Distance distributions for the two binding pockets (red line corresponds to the first pocket, whereas black line refers to the second pocket). 


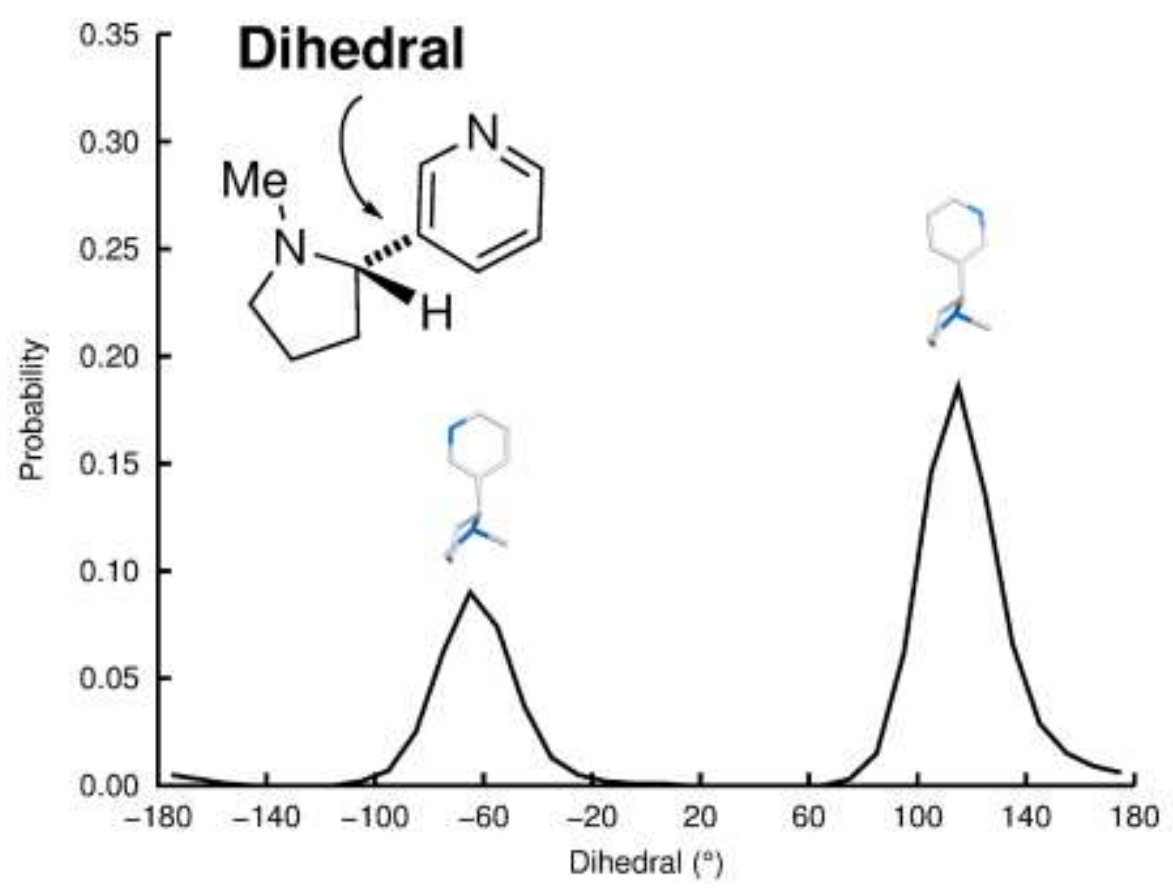

Figure S9- Dihedral (between the pyridine and pyrrolidine rings) angle distribution for nicotine in equilibrium MD simulations. This histogram reflects the dihedral distribution for nicotine in the two binding pockets. Similar distribution profiles are observed for the individual binding pockets, with the preferred binding mode around $115^{\circ}$. 


\section{Nicotine-induced conformational changes}

The $\mathrm{C}_{\alpha}$ positional deviations between the APO and the NCT systems were calculated as a function of the residue number for the last $10 \mathrm{~ns}$ of simulation in order to identify nicotine-induced conformational changes (Figures S10-S11). The final deviation values correspond to the average obtained over all 10 APO $\times 10$ NCT pairs of trajectories. The $\mathrm{C}_{\alpha}$ positional deviations were, then, mapped onto the average APO structure in order to identify the tridimensional location of the residues undergoing the largest rearrangements (Figures S12-S14).
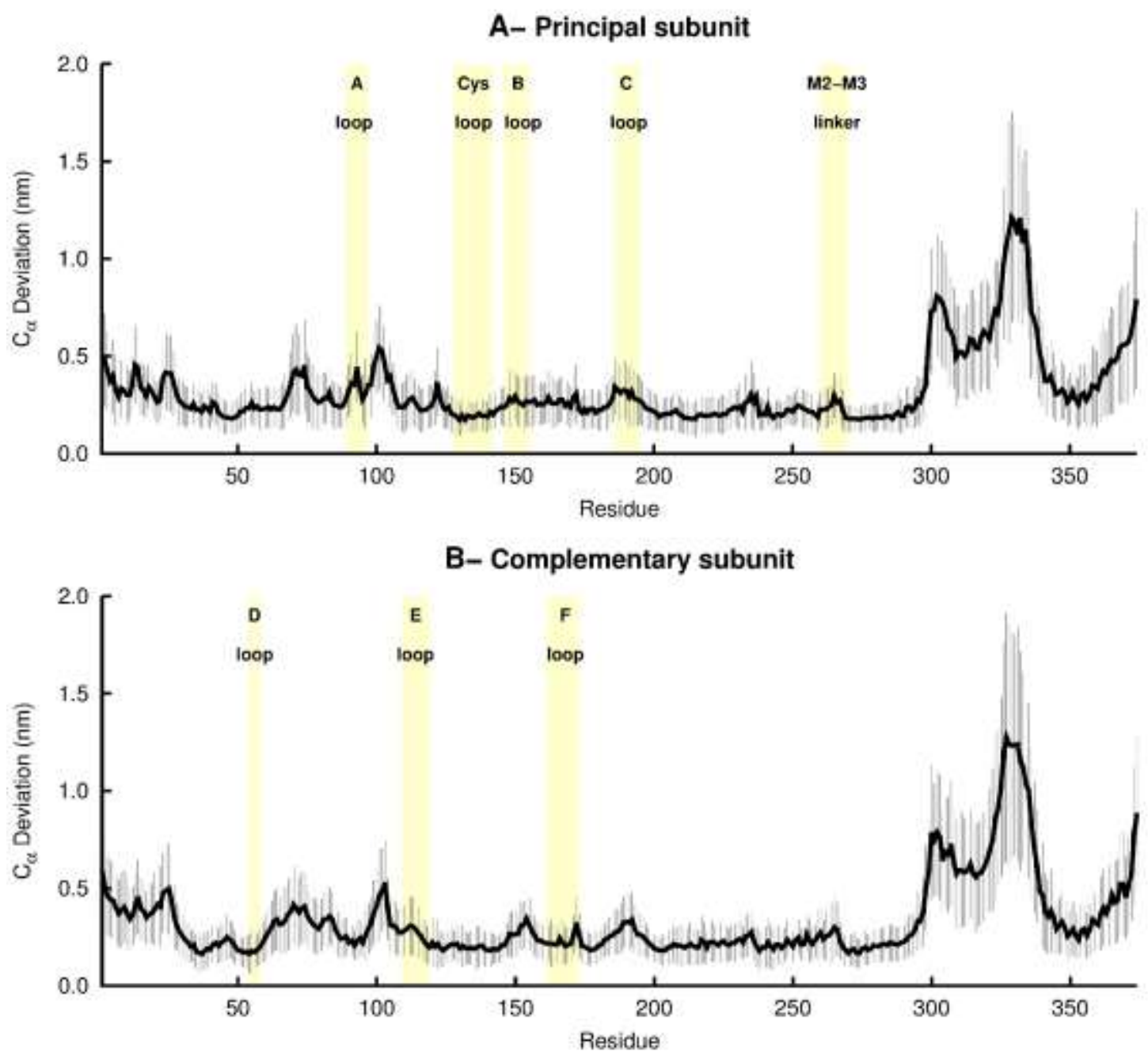

Figure S10. Average $C_{a}$ positional deviation between the APO and the NCT systems for the two subunits forming the first binding pocket. The average deviation was determined from all 100 combinations (resulting from the 10 APO $\times 10$ NCT pairs of trajectories) of $\mathrm{C}_{\alpha}$ RMSD between the average structures of the two systems. The vertical lines represent the standard deviation of the mean. The positions of some essential structural motifs are highlighted in light yellow. Note that despite some 
differences in amplitude, similar structural rearrangements are observed in the two binding pockets with largest differences not only in loops B and C and in the extracellular selectivity filter (the region after loop $\mathrm{A}$ and the region before the loop E) but also at the interface between the ECD and the TMDs, namely in the Cys and F loops and in the M2-M3 linker.
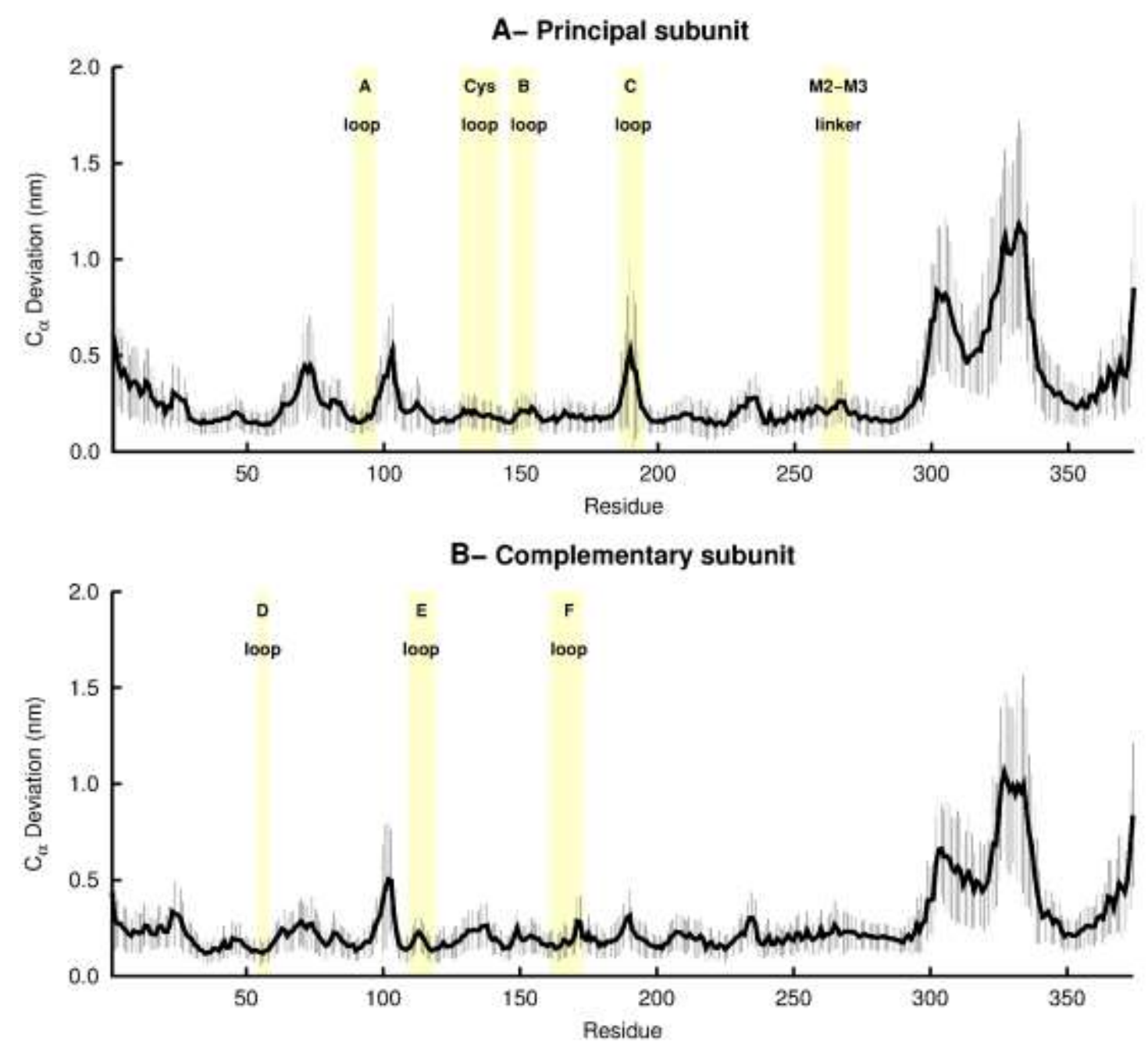

Figure S11. Average $C_{a}$ positional deviation between the APO and the NCT systems for the two subunits that form the second binding pocket. For details, see Figure S9. Note that despite some differences in amplitude, similar structural rearrangements are observed in the two binding pockets with largest differences not only in loops B and $\mathrm{C}$ and in the extracellular selectivity filter (the region after loop $\mathrm{A}$ and the region before the loop E) but also at the interface between the ECD and the TMDs, namely in the Cys and F loops and in the M2-M3 linker. 

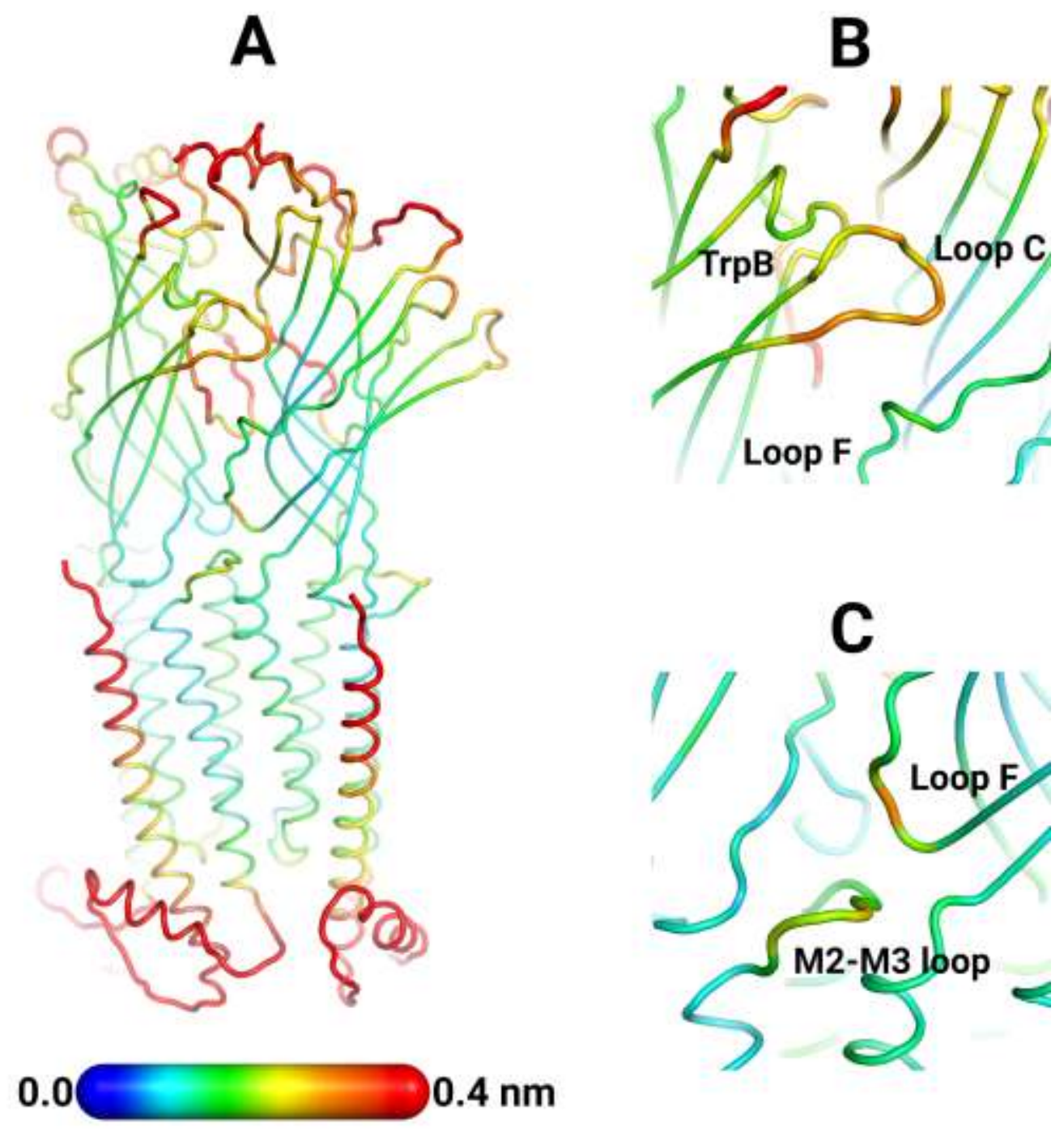

Figure S12. Nicotine-induced conformational changes in the first binding pocket. A. Comparison between the NCT and the APO systems for the first binding pocket. B. Detailed view of the binding pocket. C. Detailed view of the ECD-TMD interface. The structure colours and the cartoon thickness in this image are related to the average $\mathrm{C} \alpha$ positional deviation: the red regions correspond to the residues with the largest differences between the two systems. 

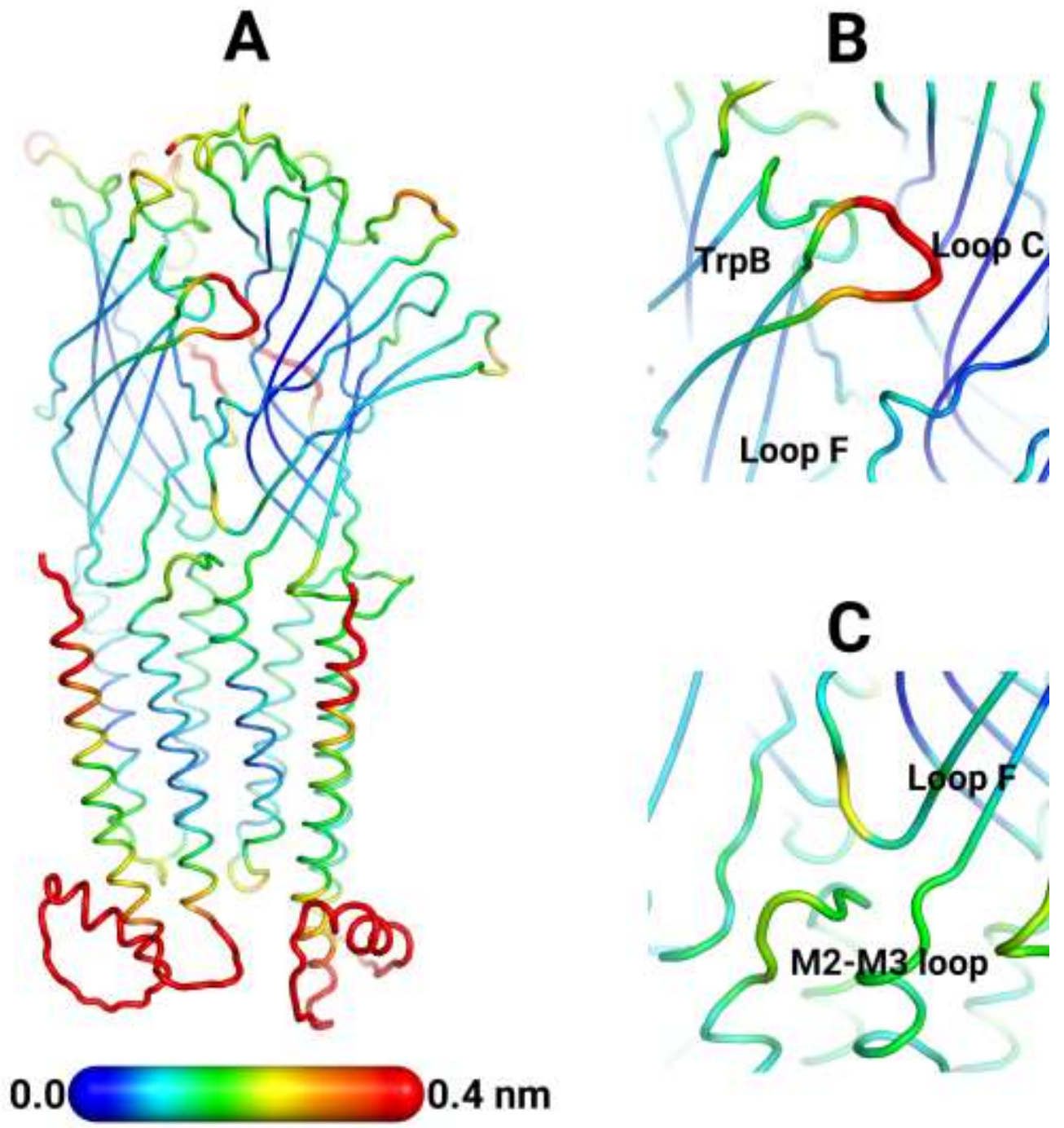

Figure S13. Nicotine-induced conformational changes at the second binding pocket. A. Comparison between the NCT and the APO systems for the second binding pocket. B. Detailed view of the binding pocket. C. Detailed view of the ECD-TMD interface. For details, see Figure S12. 


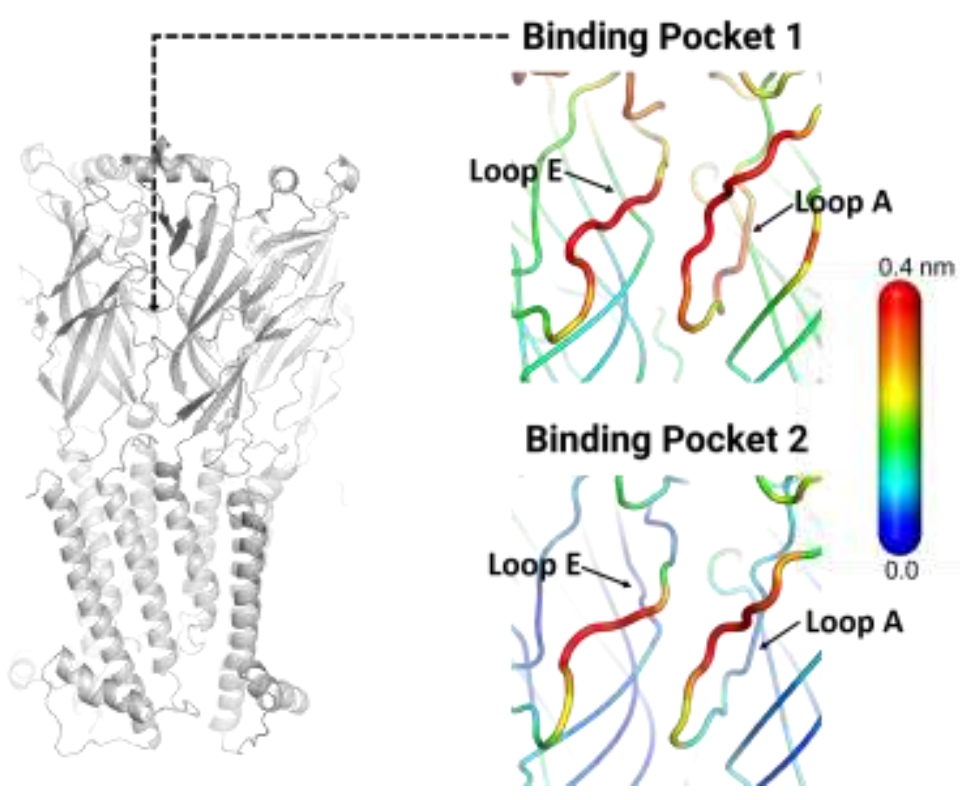

Figure S14. Zoom view of the nicotine-induced conformational changes in the extracellular vestibule in the first (top right image) and second (bottom right image). For details, see Figure S12. Note that the residues located next to loop A and E (right-side images) are located in the second layer of residues surrounding the binding pockets and are part of the extracellular selectivity filter. Mutations in these regions influence ion conductance in pLGICs (e.g. ${ }^{42,43}$ ). 


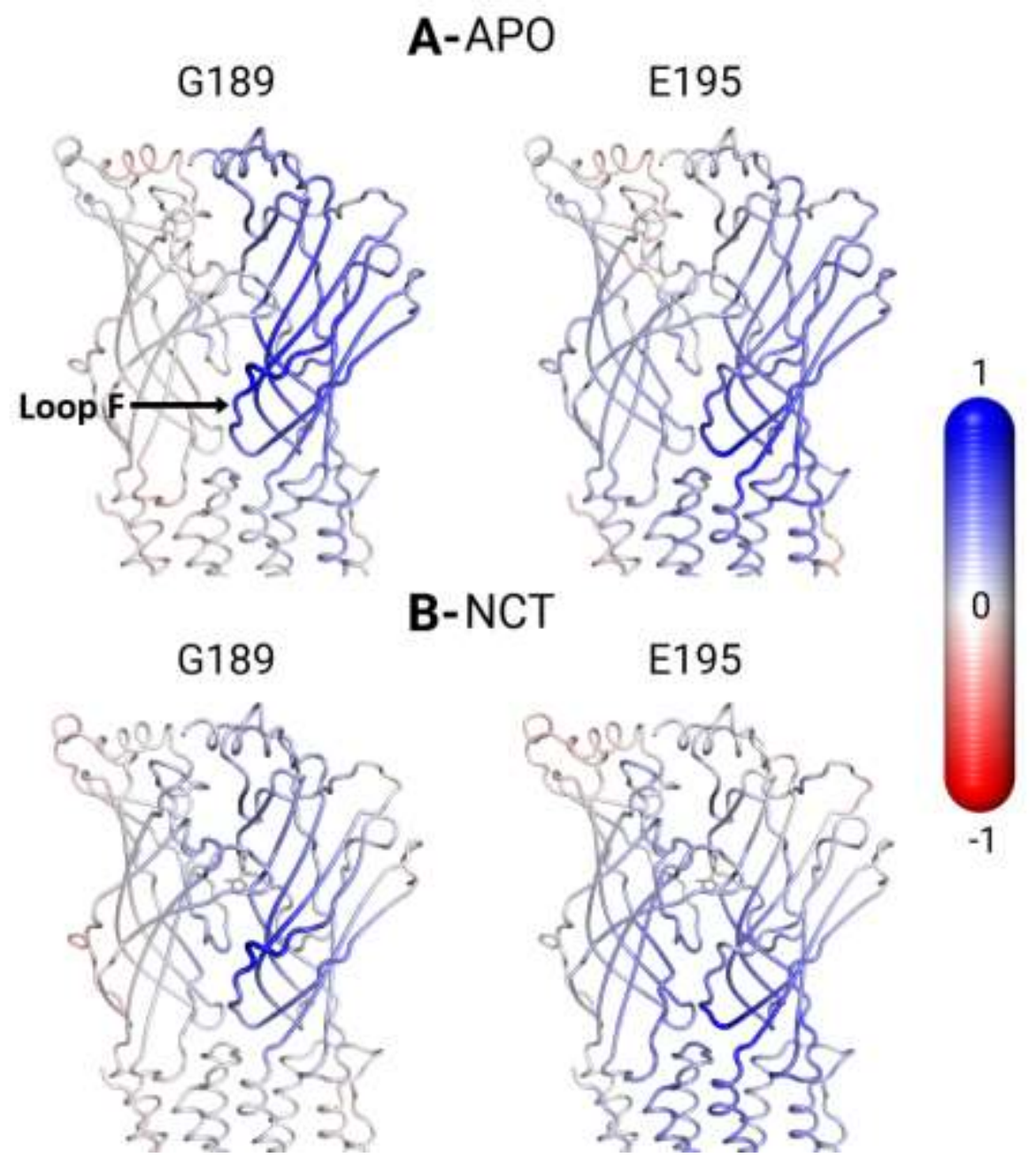

Figure S15. Statistical correlations for loop $\mathbf{F}$ in the first binding pocket. On the left side of the image, the correlations between the $\mathrm{C} \alpha$ atom of G189 (located in the top part of loop F) in the complementary subunits and all the remaining $\mathrm{C} \alpha$ atoms are shown. On the right side, the correlations between the $\mathrm{C} \alpha$ atom of E195 (located in the lower part of loop F) and all the remaining $\mathrm{C} \alpha$ atoms are mapped. In this image, the atoms that systematically move along the opposite/same direction as G189 (in the left side) or E195 (in the right side) have a correlation value of $-1 / 1$, whereas those whose movements are uncorrelated present value of 0 . In this image, the correlations are mapped on the average NCT structure (the average structure was obtained from the last $450 \mathrm{~ns}$ of simulation over all 10 replicates). 


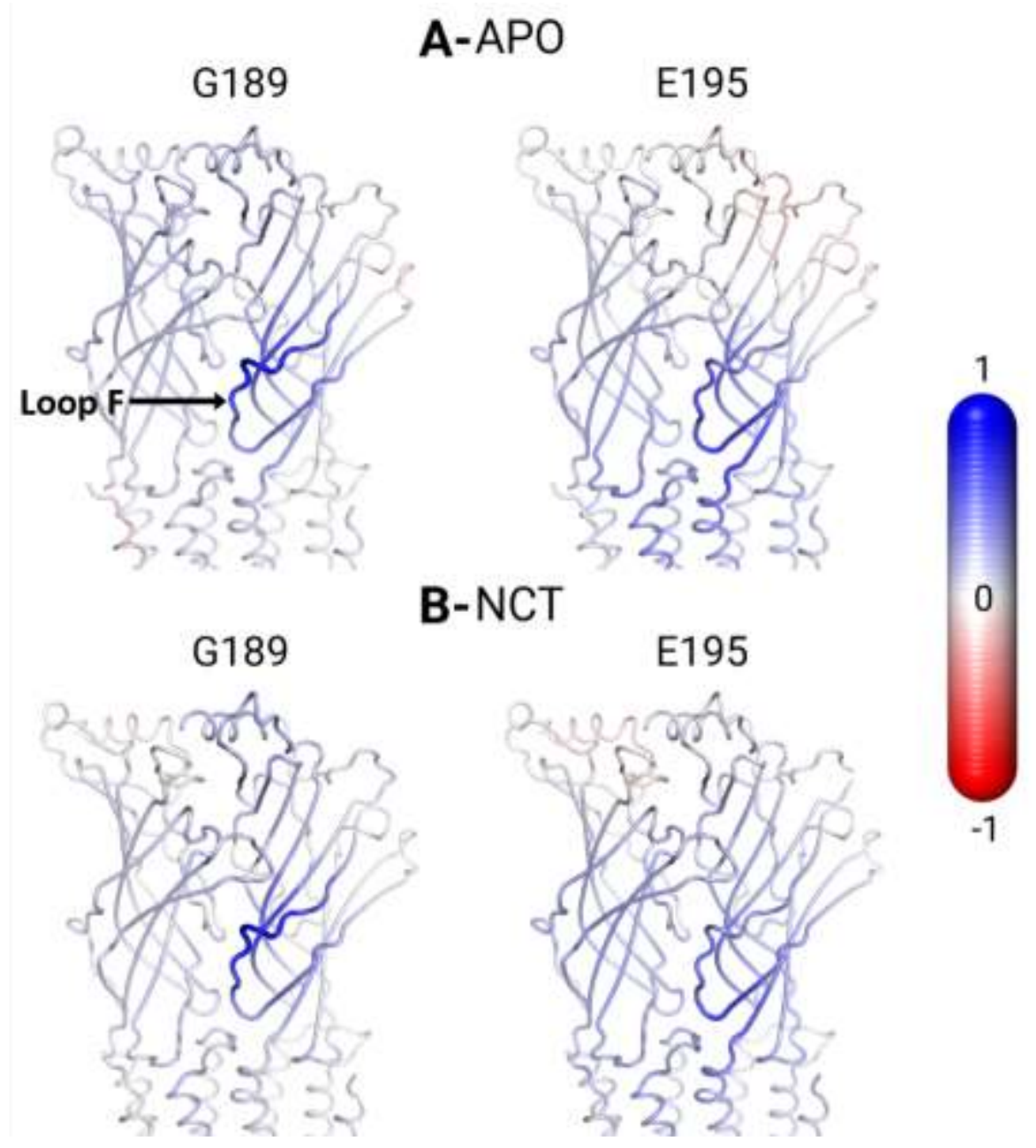

Figure S16. Statistical correlations for loop $\mathbf{F}$ in the second binding pocket. For details, see Figure S15. 


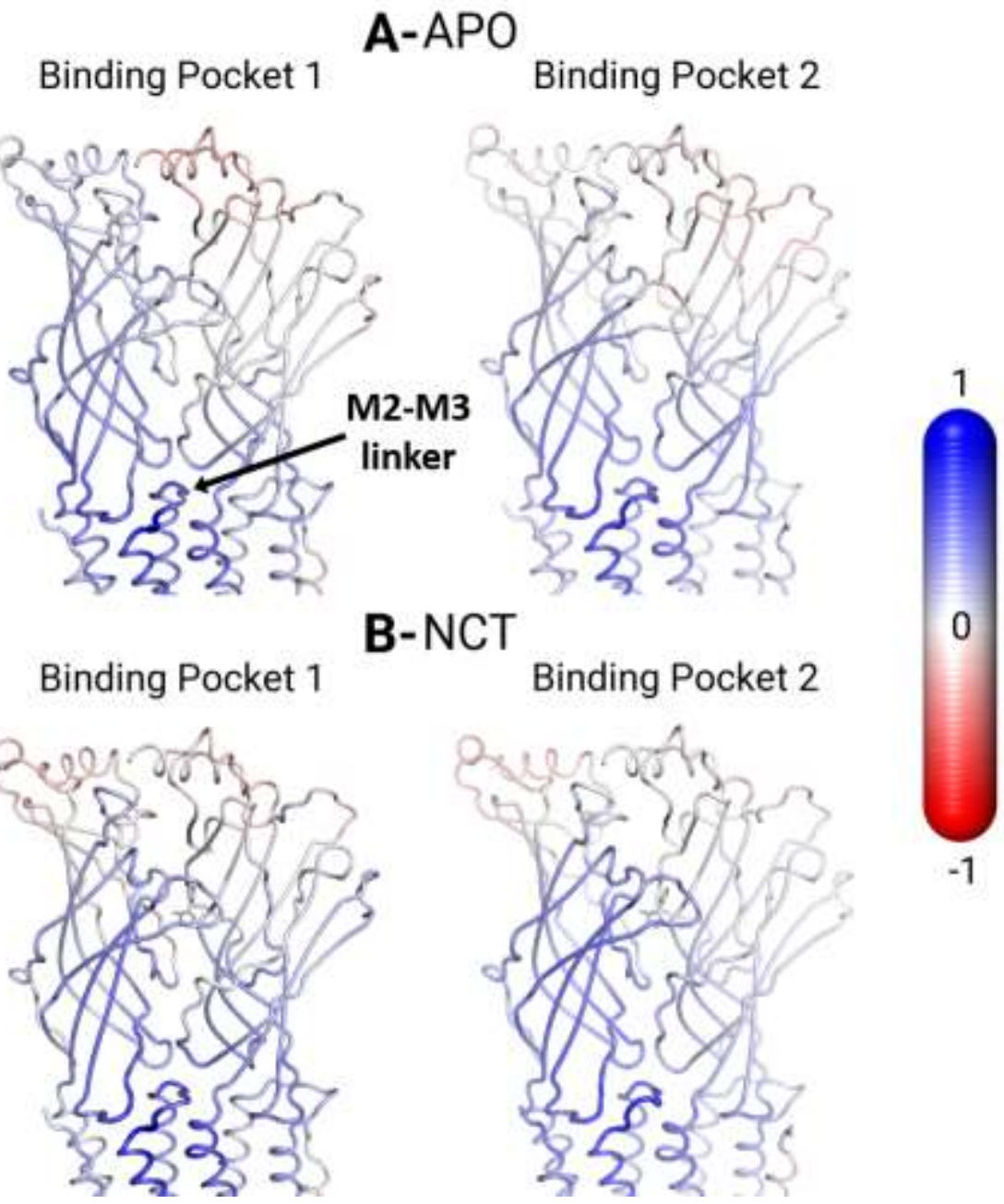

Figure S17. Statistical correlations for the M2-M3 linker. The correlations between the C $\alpha$ atom of V290 (located in the M2-M3 linker) in the principal subunits and all the remaining C $\alpha$ atoms are shown. In this image, the atoms that systematically move along the opposite/same direction as V290 have a correlation value of $-1 / 1$, respectively. In this image, the correlations are mapped on the average NCT structure (the average structure was obtained from the last $450 \mathrm{~ns}$ of simulation over all 10 replicates). 


\section{Nonequilibrium analysis}

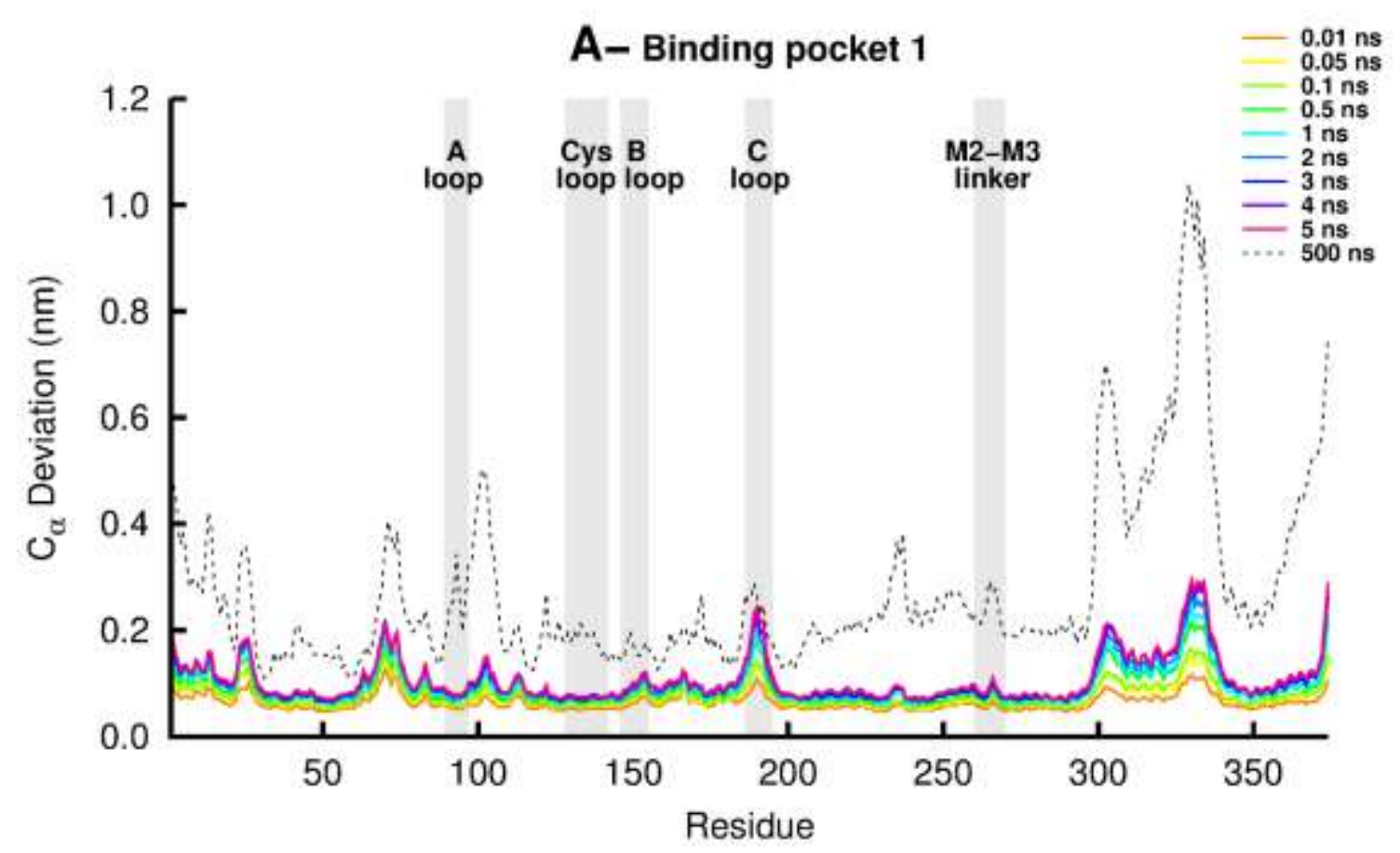

B- Binding pocket 2

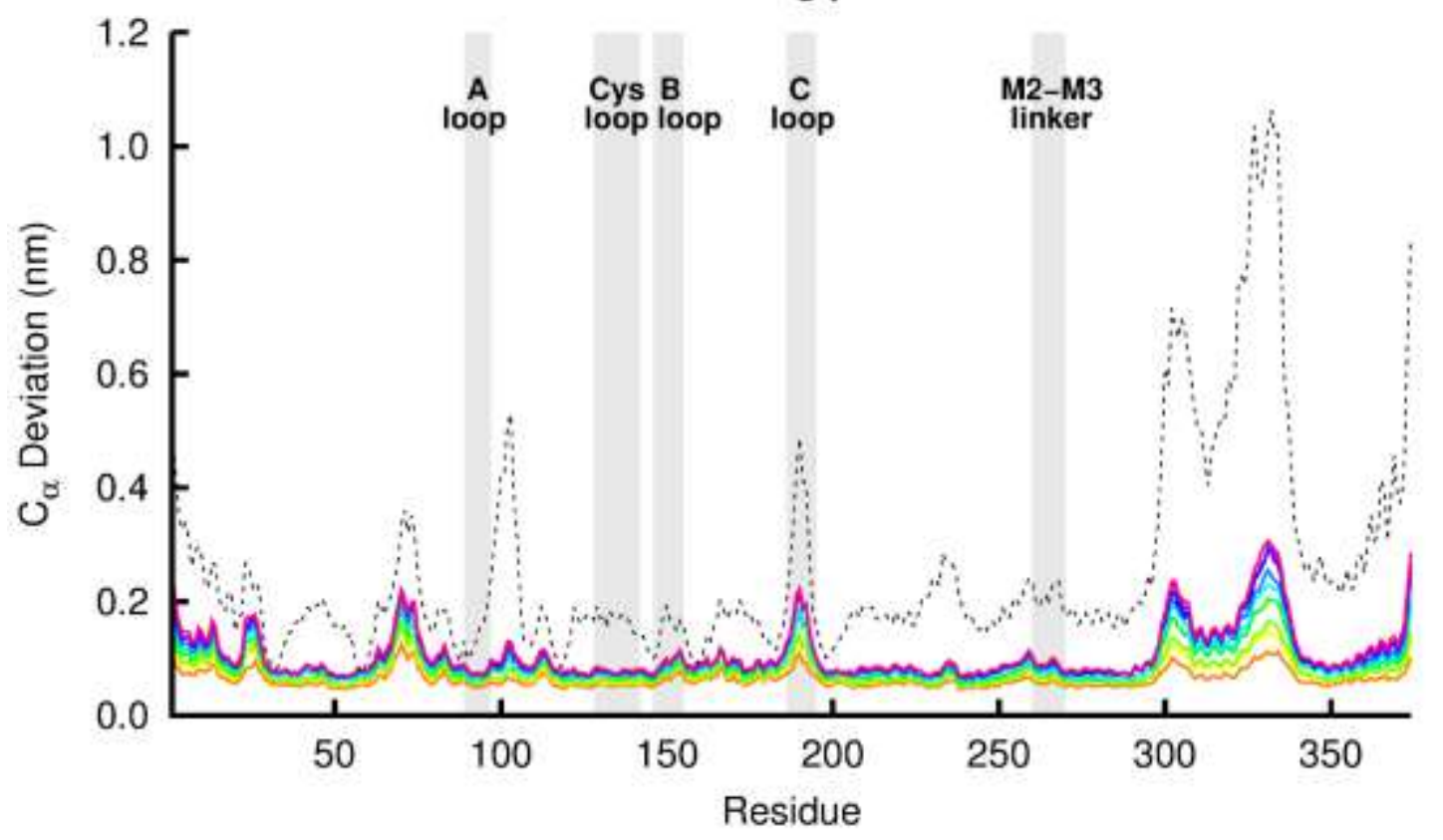

Figure S18. Average $C_{\alpha}$-positional deviation for the principal subunits in the 5 ns after the removal of nicotine from the binding pockets. The average deviation was calculated using the Kubo-Onsager approach $^{30-32}$ for the comparison of the 450 short APO simulations with the NCT simulations. The positions of the structural motifs that form the binding pockets are highlighted in grey. The deviation obtained from the long simulations (500 ns) is also shown (dotted black line) for comparison, and it 
corresponds to the average $\mathrm{C}_{\alpha}$ positional deviation plotted in Figure S10 and S11. Note that the deviations observed after $5 \mathrm{~ns}$ are far from the scale of the rearrangements observed in the long equilibrium simulations, especially regarding the A, B and Cys loops. Note also the equivalent response of the two binding pockets to nicotine annihilation, with a similar order of events associated with signal propagation being observed. 

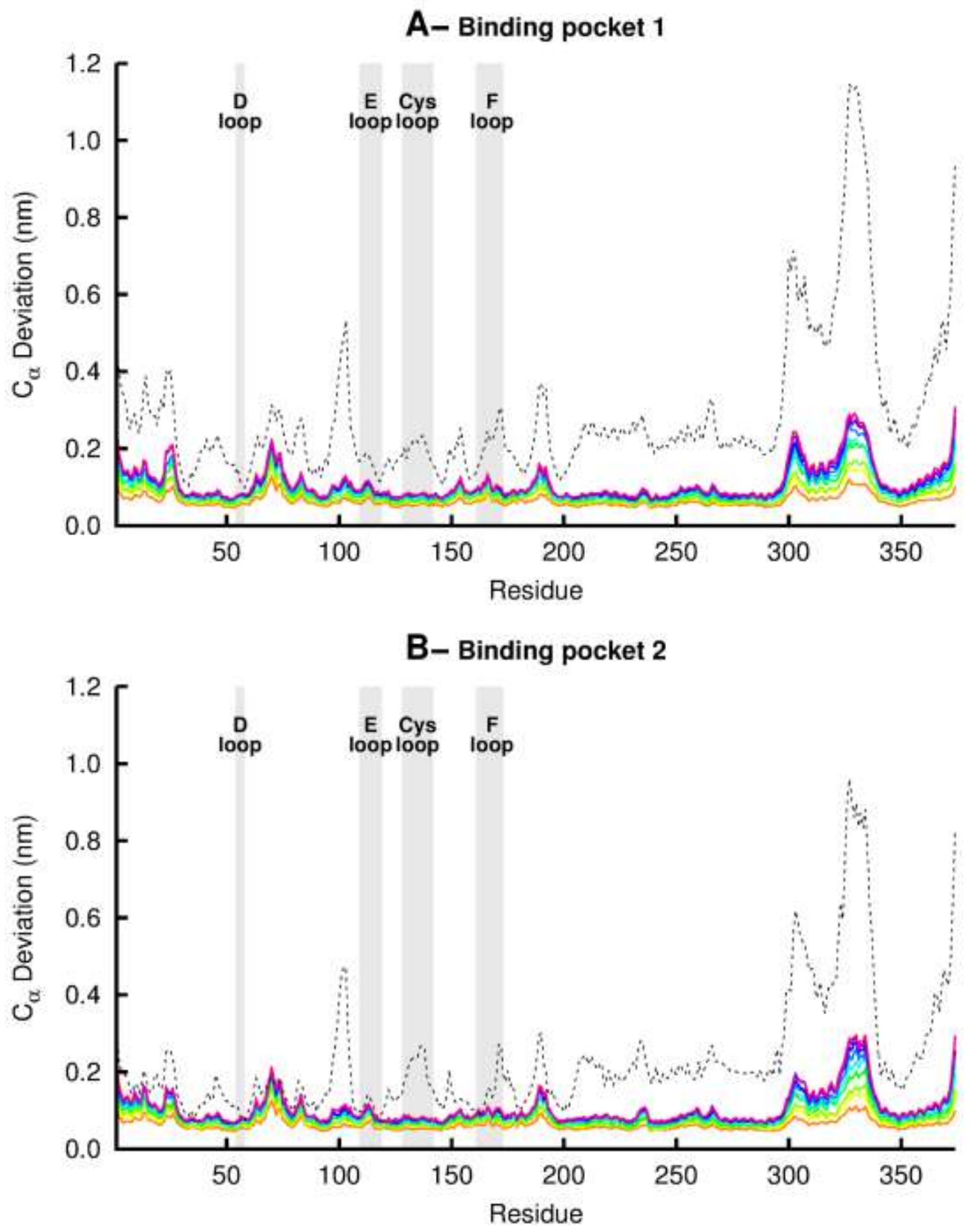

Figure S19. Average $C_{\alpha}$-positional deviation for the complementary subunits in the 5 ns after nicotine's removal from the binding pockets. The average deviation was calculated using the KuboOnsager approach ${ }^{30-32}$ for the comparison of the 450 short APO simulations with the NCT simulations. The positions of the structural motifs that form the binding pockets are highlighted in grey. The deviation obtained from the long simulations (500 ns) is also shown (dotted black line) for comparison, and it corresponds to the average $\mathrm{C}_{\alpha}$ positional deviation plotted in Figure S10 and S11. Note also the equivalent response of the two binding pockets to nicotine annihilation with a similar order of events associated with signal propagation being observed. 


\section{$0.00 \mathrm{~ns}$}

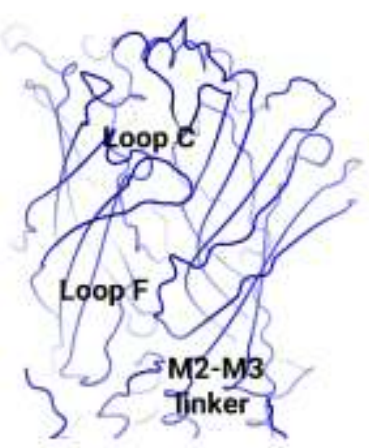

$1 \mathrm{~ns}$

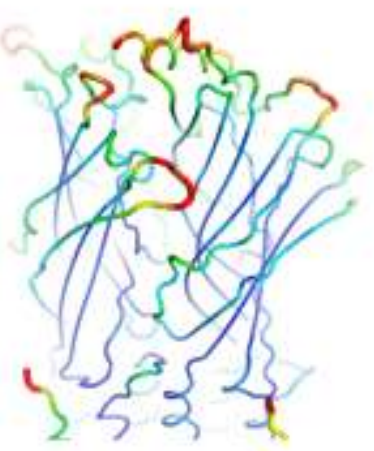

\section{$0.05 \mathrm{~ns}$}

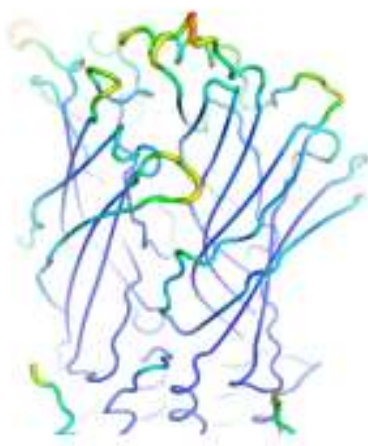

3 ns

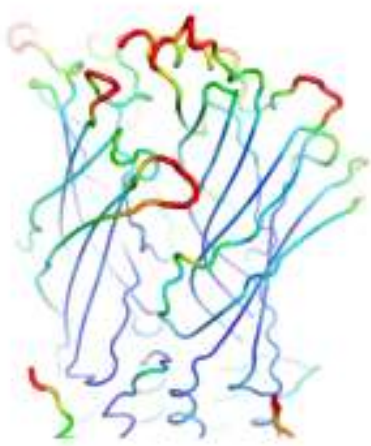

Ca Deviation $(\mathrm{nm})$

\section{$0.5 \mathrm{~ns}$}

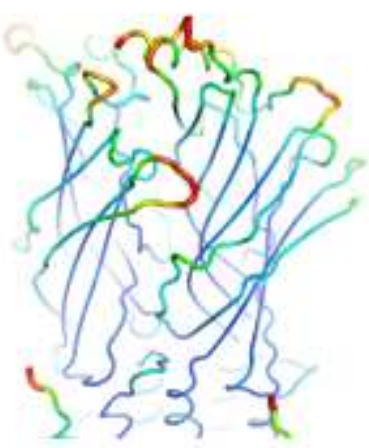

$5 \mathrm{~ns}$

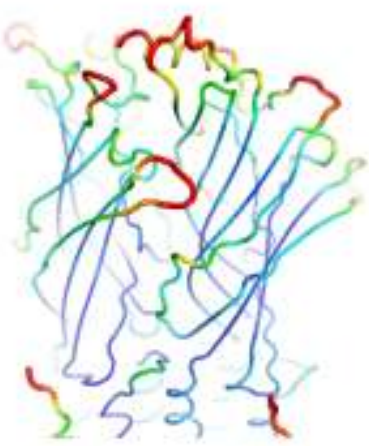

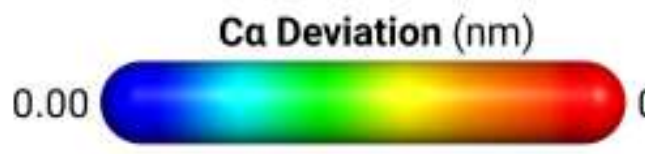

0.18

Figure S20. Mapping of the average Co-positional deviation in the $5 \mathrm{~ns}$ following nicotine removal from the first binding pocket. The $\mathrm{C}_{\alpha}$ deviation between the short APO and the NCT simulations at specific times $(0,0.05,0.5,1,3$ and $5 \mathrm{~ns})$ after removal of the ligand were calculated as a function of the residue number. The final deviation values correspond to the average obtained over all 450 pairs of simulations (see Figure S18-S19). In this image, the average deviations are mapped on the average APO structure, using the colour scheme presented in the scale. 
$0.00 \mathrm{~ns}$

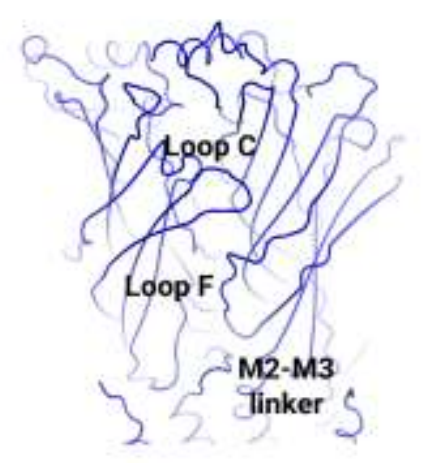

$1 \mathrm{~ns}$

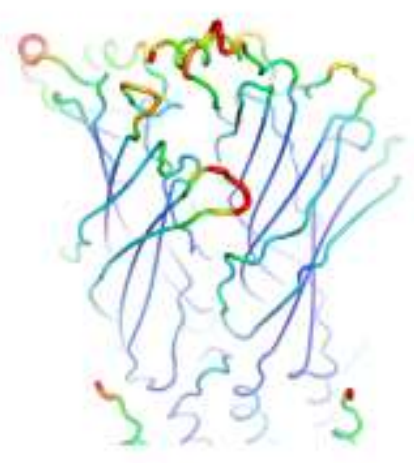

$0.05 \mathrm{~ns}$

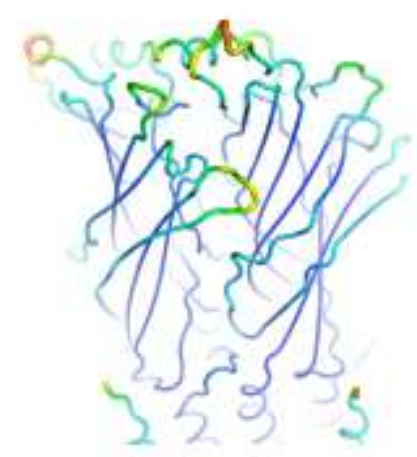

$3 \mathrm{~ns}$

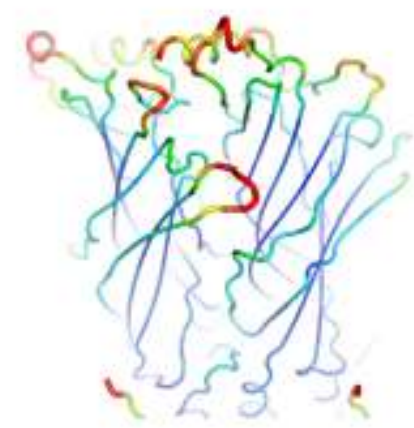

Ca Deviation (nm)

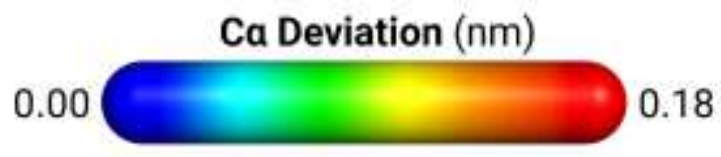

Figure S21. Mapping of the average Co-positional deviation in the 5 ns following the removal of nicotine from the second binding pocket. For details, see Figure S20. 

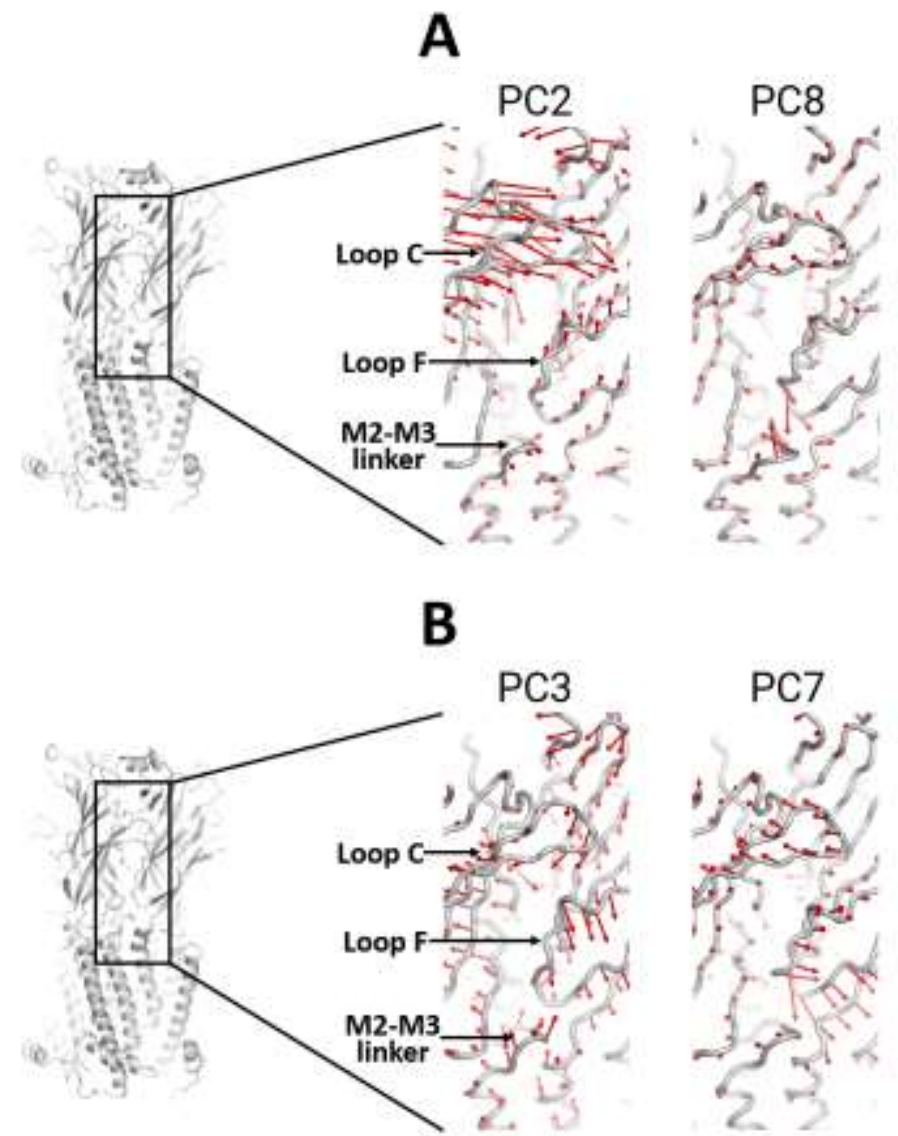

Figure S22. Concerted motions in the binding pocket region and at the ECD:TMD interface in the equilibrium APO (A) and nonequilibrium (B) simulations. For the PCA of the equilibrium simulations, all 10 replicates were combined whereas for the nonequilibrium simulations, all 450 replicates were combined before the analysis. Each PCA trajectory contains one conformation per nanosecond per replicate using the $\mathrm{C}_{\alpha}$ atoms of the protein (except for the $\mathrm{N}$ - and $\mathrm{C}$-terminal, and the loop between TMD M3 and M4). Note that in the equilibrium simulations (panel A), the individual motions associated with the signal propagation are also sampled (e.g. note the correlated motions between loop C and the top region of loop F or between the lower region of loop F and the M2-M3 linker in the principal components 2 and 8 , respectively). On the nonequilibrium simulations, a concerted motion connecting the binding pocket (through loops $\mathrm{C}$ and F) and the TMDs (via the M2-M3 linker) can be observed (see principal components 3 and 7 in the panel B). It is also noteworthy that the PCA of the nonequilibrium simulations shows the same collective motions of the C-loop, the F loop and the M2-M3 linker as revealed by the Kubo-Onsager analysis. Nonetheless, the PCA analysis loses the temporal order of events that is revealed by the Kubo-Onsager analysis.

\section{Movie 1}




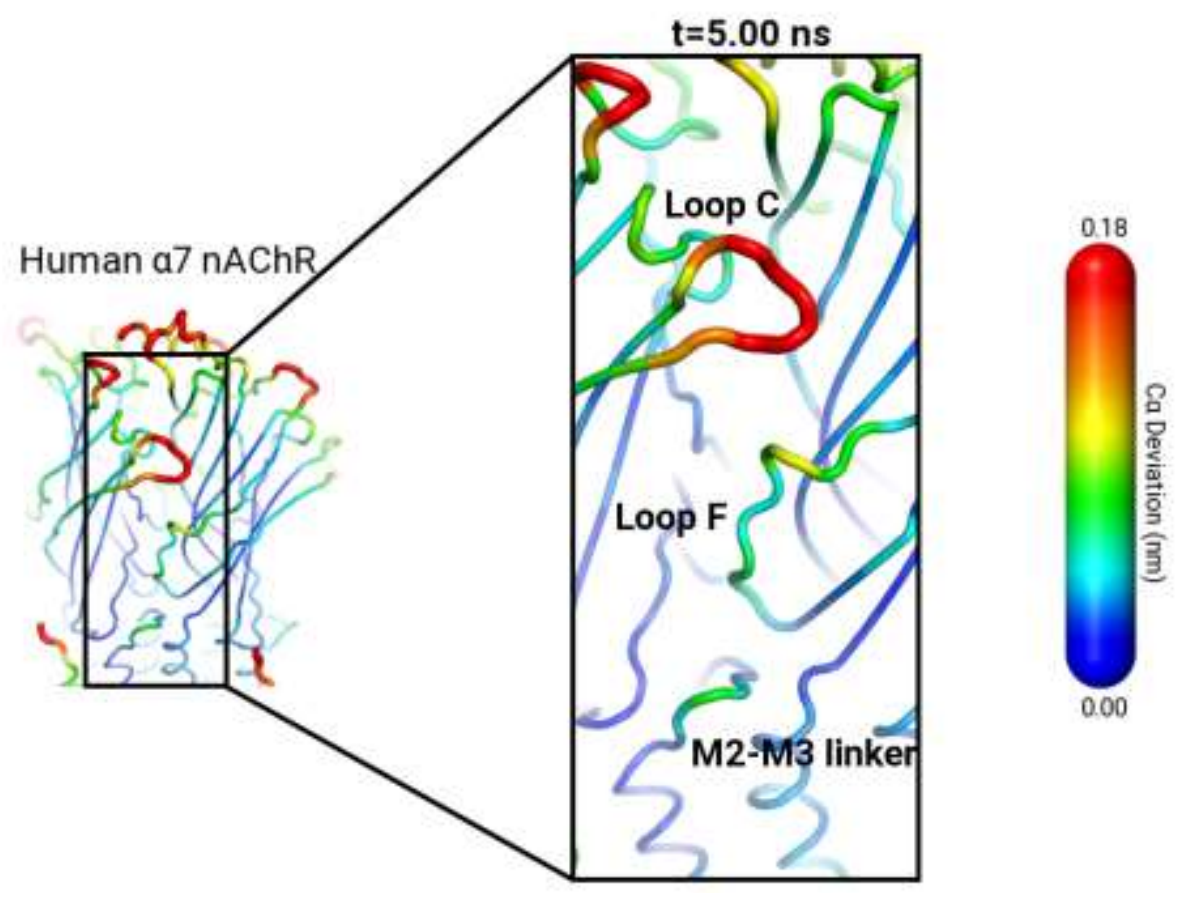

Movie 1. Signal propagation in the human $\alpha 7 \mathrm{nAChR}$ (movie1.avi). This movie depicts signal propagation along the $5 \mathrm{~ns}$ after the deletion of nicotine from the first binding pocket in the human $\alpha 7$ nAChR subtype.

Movie 2

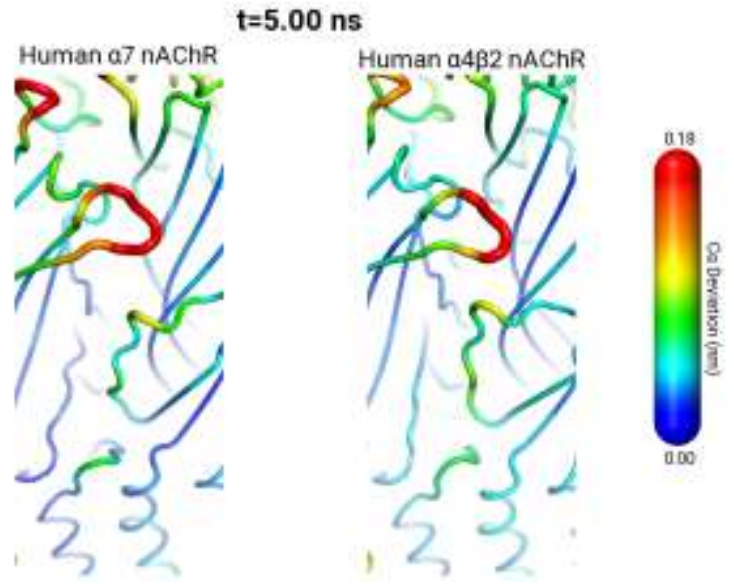


Movie 2- Signal propagation in the human $\alpha 7$ and $\alpha 4 \beta 2$ nAChR subtypes (movie2.avi). Note that despite the differences in propagation rates, the structural motifs involved, as well as the sequence of events associated with the first steps of signal transmission, are the same for both subtypes.

Movie 3

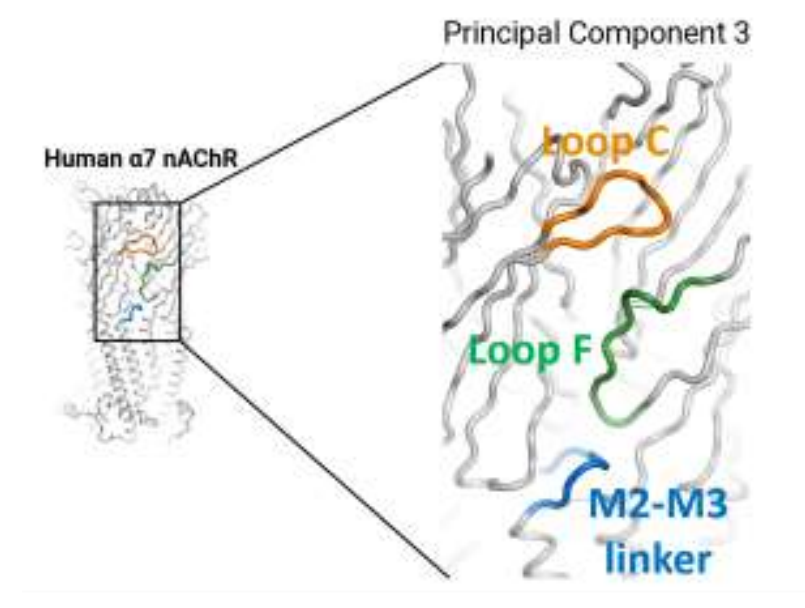

Movie 3. Concerted motion (principal component 3) between the binding pocket and the TMDs observed in the nonequilibrium simulations of the human $\alpha 7 \mathrm{nAChR}$ (movie3.avi). For details regarding the PCA performed, see Figure S22.

\section{References}

1. Morales-Perez, C. L.; Noviello, C. M.; Hibbs, R. E., X-ray structure of the human alpha 4 beta 2 nicotinic receptor. Nature 2016, 538, 411-415.

2. Bateman, A.; Martin, M.; O'Donovan, C.; Magrane, M.; Alpi, E.; Antunes, R.; Bely, B.; Bingley, M.; Bonilla, C.; Britto, R.; Bursteinas, B.; Bye-A-Jee, H.; Cowley, A.; Da Silva, A.; De Giorgi, M.; Dogan, T.; Fazzini, F.; Castro, L.; Figueira, L.; Garmiri, P.; Georghiou, G.; Gonzalez, D.; Hatton-Ellis, E.; Li, W.; Liu, W.; Lopez, R.; Luo, J.; Lussi, Y.; MacDougall, A.; Nightingale, A.; Palka, B.; Pichler, K.; Poggioli, D.; Pundir, S.; Pureza, L.; Qi, G.; Rosanoff, S.; Saidi, R.; Sawford, T.; Shypitsyna, A.; Speretta, E.; Turner, E.; Tyagi, N.; Volynkin, V.; Wardell, T.; Warner, K.; Watkins, X.; Zaru, R.; Zellner, H.; Xenarios, I.; Bougueleret, L.; Bridge, A.; Poux, S.; Redaschi, N.; Aimo, L.; Argoud-Puy, G.; Auchincloss, A.; Axelsen, K.; Bansal, P.; Baratin, D.; Blatter, M.; Boeckmann, B.; Bolleman, J.; Boutet, E.; Breuza, L.; Casal-Casas, C.; de Castro, E.; Coudert, E.; Cuche, B.; Doche, M.; Dornevil, D.; Duvaud, S.; Estreicher, A.; Famiglietti, L.; Feuermann, M.; Gasteiger, E.; Gehant, S.; Gerritsen, V.; Gos, A.; Gruaz-Gumowski, N.; Hinz, U.; Hulo, C.; Jungo, F.; Keller, G.; Lara, V.; Lemercier, P.; 
Lieberherr, D.; Lombardot, T.; Martin, X.; Masson, P.; Morgat, A.; Neto, T.; Nouspikel, N.; Paesano, S.; Pedruzzi, I.; Pilbout, S.; Pozzato, M.; Pruess, M.; Rivoire, C.; Roechert, B.; Schneider, M.; Sigrist, C.; Sonesson, K.; Staehli, S.; Stutz, A.; Sundaram, S.; Tognolli, M.; Verbregue, L.; Veuthey, A.; Wu, C.; Arighi, C.; Arminski, L.; Chen, C.; Chen, Y.; Garavelli, J.; Huang, H.; Laiho, K.; McGarvey, P.; Natale, D.; Ross, K.; Vinayaka, C.; Wang, Q.; Wang, Y.; Yeh, L.; Zhang, J.; Consortium, U., UniProt: the universal protein knowledgebase. Nucleic Acids Res. 2017, 45 (D1), D158-D169.

3. McWilliam, H.; Li, W.; Uludag, M.; Squizzato, S.; Park, Y.; Buso, N.; Cowley, A.; Lopez, R., Analysis Tool Web Services from the EMBL-EBI. Nucleic Acids Res. 2013, 41 (W1), W597-W600.

4. Sievers, F.; Higgins, D., Clustal Omega for making accurate alignments of many protein sequences. Protein Sci. 2018, 27 (1), 135-145.

5. Altschul, S.; Madden, T.; Schaffer, A.; Zhang, J.; Zhang, Z.; Miller, W.; Lipman, D., Gapped BLAST and PSI-BLAST: a new generation of protein database search programs. Nucleic Acids Res. 1997, 25 (17), 3389-3402.

6. Sali, A.; Potterton, L.; Yuan, F.; Vanvlijmen, H.; Karplus, M., Evaluation of Comparative Protein Modeling by Modeler. Proteins 1995, 23, 318-326.

7. Laskowski, R. A.; Macarthur, M. W.; Moss, D. S.; Thornton, J. M., Procheck - a Program to Check the Stereochemical Quality of Protein Structures. J. Appl. Crystallogr. 1993, 26, 283-291.

8. Bouzat, C.; Rayes, D.; De Rosa, M.; Sine, S., Electrical Fingerprinting Reveals Agonist Binding Sites Required for Activation of Homo-pentameric Cys-loop Receptors. Biophys. J. 2009, 96 (3), 167A-167A.

9. Nys, M.; Kesters, D.; Ulens, C., Structural insights into Cys-loop receptor function and ligand recognition. Biochem. Pharmacol. 2013, 86, 1042-1053.

10. Oliveira, A. S. F.; Shoemark, D. K.; Campello, H. R.; Gallagher, T.; Sessions, R. B.; Mulholland, A. J., Identification of the initial steps in signal transduction in the $\alpha 4 \beta 2$ nicotinic receptor: insights from equilibrium and nonequilibrium simulations. Structure 2019, 27, 1171-1183.

11. Bashford, D.; Karplus, M., pKa's of ionizable groups in proteins: atomic detail from a continuum electrostatic model. Biochemistry 1990, 29, 10219-25.

12. Bashford, D.; Gerwert, K., Electrostatic calculations of the $\mathrm{pKa}$ values of ionizable groups in bacteriorhodopsin. J. Mol. Biol. 1992, 224, 473-486.

13. Bashford, D., An object-oriented programming suite for electrostatic effects in biological molecules. In Scientific Computing in Object-Oriented Parallel Environments, Ishikawa, Y.; Oldehoeft, R. R.; Reynders, J. V. W.; Tholburn, M., Eds. ISCOPE97, Springer: Berlin, 1997; Vol. 1343, pp 233-240.

14. Baptista, A. M.; Soares, C. M., Some theoretical and computational aspects of the inclusion of proton isomerism in the protonation equilibrium of proteins. J. Phys. Chem. B 2001, 105, 293-309.

15. Yang, S. S.; Smetena, I., Evaluation of Capillary Electrophoresis for the Analysis of Nicotine and Selected Minor Alkaloids from Tobacco. Chromatographia 1995, 40, 375-378.

16. Schmidt, T. H.; Kandt, C., LAMBADA and InflateGRO2: Efficient Membrane Alignment and Insertion of Membrane Proteins for Molecular Dynamics Simulations. $J$. Chem. Inf. Model. 2012, 52, 2657-2669.

17. Jorgensen, W. L.; Chandrasekhar, J.; Madura, J. D.; Impey, R. W.; Klein, M. L., Comparison of Simple Potential Functions for Simulating Liquid Water. J. Chem. Phys. 1983, 79, 926-935. 
18. Abraham, M. J.; Murtola, T.; Schulz, R.; Pall, S.; Smith, J. C.; Hess, B.; Lindahl, E., GROMACS: High performance molecular simulations through multi-level parallelism from laptops to supercomputers. SoftwareX 2015, 1-2, 19-25.

19. Lindorff-Larsen, K.; Piana, S.; Palmo, K.; Maragakis, P.; Klepeis, J. L.; Dror, R. O.; Shaw, D. E., Improved side-chain torsion potentials for the Amber ff99SB protein force field. Proteins 2010, 78, 1950-1958.

20. Jambeck, J. P. M.; Lyubartsev, A. P., An Extension and Further Validation of an All-Atomistic Force Field for Biological Membranes. J. Chem. Theory Comput. 2012, 8, 2938-2948.

21. Jambeck, J. P. M.; Lyubartsev, A. P., Derivation and Systematic Validation of a Refined All-Atom Force Field for Phosphatidylcholine Lipids. J. Phys. Chem. B 2012, 116, 3164-3179.

22. Campello, H. R.; Del Villar, S. G.; Honraedt, A.; Viñas, T. M.; Oliveira, A. S. F.; Ranaghan, K. E.; Shoemark, D. K.; Bermudez, I.; Gotti, C.; Sessions, R. B.; Mulholland, A. J.; Wonnacott, S.; Gallagher, T., Unlocking Nicotinic Selectivity via Direct C-H Functionalisation of (-)-Cytisine. Chem 2018, 4, 1710-1725.

23. Bussi, G.; Donadio, D.; Parrinello, M., Canonical sampling through velocity rescaling. J. Chem. Phys. 2007, 126, 014101.

24. Berendsen, H.; Postma, J.; van Gunsteren, W.; Dinola, A.; Haak, J., Molecular dynamics with coupling to an external bath. J. Chem. Phys. 1984, 81, 3684-3690.

25. Essmann, U.; Perera, L.; Berkowitz, M. L., A smooth particle mesh Ewald method. J. Chem. Phys. 1995, 103, 8577-8593.

26. Allen, M. P.; Tildesley, D. J., Computer simulation of liquids. Clarendon Press: Oxford, UK, 1987.

27. Hess, B.; Bekker, H.; Berendsen, H. J. C.; Fraaije, J. G. E. M., LINCS: a linear constraint solver for molecular simulations. J. Comput. Chem. 1997, 18, 1463-1472.

28. Miyamoto, S.; Kollman, P. A., SETTLE: An Analytical Version of the SHAKE and RATTLE Algorithms for Rigid Water Models. J. Comput. Chem. 1992, 13, 952962.

29. Nemecz, A.; Prevost, M. S.; Menny, A.; Corringer, P. J., Emerging Molecular Mechanisms of Signal Transduction in Pentameric Ligand-Gated Ion Channels. Neuron 2016, 90, 452-470.

30. Ciccotti, G.; Jacucci, G.; McDonald, I. R., Thought-Experiments by Molecular Dynamics. J. Stat. Phys. 1979, 21, 1-21.

31. Ciccotti, G., Molecular dynamics simulation of non equilibrium phenomena and rare dynamical events. In Computer simulation in material science, Meyer M, P. V., Ed. Kluwer Academic Publishers: 1991; pp 119-137.

32. Ciccotti, G.; Ferrario, M., Non-equilibrium by molecular dynamics: a dynamical approach. Mol. Simul. 2016, 42 (16), 1385-1400.

33. Delano, W. L., The Pymol molecular graphics system. version 0.98. Delano Scientific LLC: San Carlos, CA, USA, 2003.

34. DeLano, W. L., PyMOL molecular viewer: Updates and refinements. Abstracts of Papers of the American Chemical Society 2009, 238.

35. Roy, J.; Laughton, C. A., Long-timescale molecular-dynamics simulations of the major urinary protein provide atomistic interpretations of the unusual thermodynamics of ligand binding. Biophys. J. 2010, 99, 218-226.

36. Garton, M.; Laughton, C., A comprehensive model for the recognition of human telomeres by TRF1. J. Mol. Biol. 2013, 425, 2910-2921. 
37. Ng, H. W.; Laughton, C. A.; Doughty, S. W., Molecular dynamics simulations of the adenosine A2a receptor: structural stability, sampling, and convergence. J. Chem. Inf. Model. 2013, 53, 1168-1178.

38. Kabsch, W.; Sander, C., Dictionary of protein secondary structure: pattern recognition of hydrogen-bonded and geometrical features. Biopolymers 1983, 22, 2577 2637.

39. Dougherty, D. A., Cys-loop neuroreceptors: structure to the rescue? Chem. Rev. 2008, 108, 1642-1653.

40. Tavares Xda, S.; Blum, A. P.; Nakamura, D. T.; Puskar, N. L.; Shanata, J. A.; Lester, H. A.; Dougherty, D. A., Variations in binding among several agonists at two stoichiometries of the neuronal, alpha4beta2 nicotinic receptor. J. Am. Chem. Soc. 2012, 134, 11474-11480.

41. Van Arnam, E. B.; Dougherty, D. A., Functional probes of drug-receptor interactions implicated by structural studies: Cys-loop receptors provide a fertile testing ground. J. Med. Chem. 2014, 57, 6289-6300.

42. Hansen, S.; Wang, H.; Taylor, P.; Sine, S., An Ion Selectivity Filter in the Extracellular Domain of Cys-loop Receptors Reveals Determinants for Ion Conductance. J. Biol. Chem. 2008, 283 (52), 36066-36070.

43. Moroni, M.; Meyer, J.; Lahmann, C.; Sivilotti, L., In Glycine and GABA(A) Channels, Different Subunits Contribute Asymmetrically to Channel Conductance via Residues in the Extracellular Domain. J. Biol. Chem. 2011, 286 (15), 13414-13422. 